\title{
FAMILIES OF CUSP FORMS
}

\author{
by
}

E. Kowalski

\section{Contents}

1. Families of cusp forms and the local equidistribution problem......... 6

2. Families of $L$-functions.................................. 10

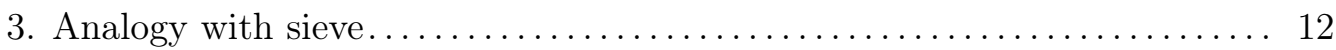

4. Examples of local equidistribution........................... 13

5. Families of cusp forms according to Sarnak's letter................ 16

6. Families of $L$-functions according to Conrey, Farmer, Keating, Rubinstein and Snaith.......................................... 17

7. Direct consequences of strong local equidistribution................ 18

8. Quantitative local equidistribution: level of decay................ 20

9. Symmetry types for the behavior of $L$-functions on the critical line..... 22

10. Symmetry types and moments of $L$-functions................... 29

11. Orthogonal symmetry types............................. 32

12. Other examples of symmetry types........................ 33

13. An example with Siegel modular forms ........................ 35

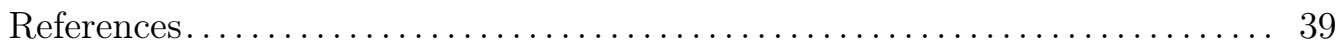

This text is a discussion of general ideas concerning families of cusp forms and their associated invariants, especially $L$-functions. The point of view is influenced by the problem of local spectral equidistribution, which makes sense in a very general setting, and seems to be a critical ingredient for systematic attempts at predicting averages of global $L$-values. The second main influence are the ideas of P. Sarnak concerning this question [20]. Among other papers that influenced the ideas here, the one of Cogdell and Michel [5] should also be mentioned.

2010 Mathematics Subject Classification. - 11F12, 11F66.

Key words and phrases. - Cups forms, L-functions, families, symmetry types. 
This text is a rather informal survey and it contains little in the way of new results. It also does not attempt to be exhaustive, and in particular the bibliography does not contain all references concerning the ideas we discuss.

Thanks to A. Venkatesh for explanations of various issues of harmonic analysis, especially with respect to Plancherel measures, and to B. Conrey for clarifying certain points in [6]. Also many thanks to A. Saha for remarks and comments about the first version of this survey. The text is presented as a list of numbered items; references to one of these are given with the sans-serif boldface font, e.g., "see 12". References to equations are in standard font within parentheses, as in "see (1)", while sections are just given as "see Section 2", and the bibliography is quoted within square brackets: "see [10]".

\section{Families of cusp forms and the local equidistribution problem}

1. For a reductive group $\mathbf{G} / k$ over a global field ${ }^{(1)} k$, with $L$-group ${ }^{L} \mathbf{G}$, we denote by $\mathcal{A}_{k}(\mathbf{G})$ or $\mathcal{A}(\mathbf{G})$ the set of cuspidal automorphic representations of $\mathbf{G}\left(\mathbf{A}_{k}\right)$ ( $\mathbf{A}_{k}$ is the ring of adèles of $k$ ). We will often drop the subscript, since $k$ is always fixed.

2. Any cusp form $\pi \in \mathcal{A}(\mathbf{G})$ can be represented as a tensor product

$$
\pi \simeq \bigotimes_{v} \pi_{v}
$$

where $v$ runs over all places of $k$, and $\pi_{v}$ is an (irreducible, unitary, admissible) representation of the group $\mathbf{G}\left(k_{v}\right)$ of points of $\mathbf{G}$ in the completion of $k$ at $v$. This is called the local component of $\pi$ at $v$.

The main point of this text is the idea that, if we have a reasonable "family" of cusp forms $\pi$, then the "local" components $\pi_{v}$, which are simpler objects, should also behave in a reasonable way for any fixed $v$, and that one should then be able to use the knowledge of such behavior to understand the family of global objects, in a local-global manner.

3. Our main concern will be $\mathbf{G}=\mathrm{GL}_{n}$ for some $n \geqslant 1$, but examples involving the symplectic group $\mathrm{Sp}_{4}$ will also occur, so we attempt a general discussion.

4. We recall, for general orientation, that if $\mathbf{G}=\mathrm{GL}_{n}$, the $L$-group is $\operatorname{simply} \mathrm{GL}_{n}(\mathbf{C})$. If $k=\mathbf{Q}, \mathbf{G}=\mathrm{GL}_{n}$ and $v$ is a finite place corresponding to a prime number $p$, then for all but finitely many $p$, the local component $\pi_{p}$ is unramified, which means that it can be parameterized uniquely by an $n$-tuple $\left(\alpha_{1}, \ldots, \alpha_{n}\right)$ of $n$ non-zero complex numbers, up to permutation, which are the Satake parameters at $p$. The Ramanujan-Petersson conjecture holds at $p$ (representation-theoretically, this means that the local component at $p$ is tempered) if and only if $\left|\alpha_{i}\right|=1$ for all $i$. In any case, however, we have $\left|\alpha_{i}\right| \leqslant \sqrt{p}$.

5. For any place $v$ of $k$, we denote by $\mathcal{A}_{v}$ or $\mathcal{A}\left(\mathbf{G}_{v}\right)$ the unitary dual of $\mathbf{G}\left(k_{v}\right)$, which is also called the "local spectrum" at $v$. Thus the local components $\pi_{v}$ of any $\pi \in \mathcal{A}(\mathbf{G})$ are elements of $\mathcal{A}_{v}$. The set $\mathcal{A}_{v}$ carries a natural topology (the Fell topology), hence a natural Borel $\sigma$ algebra. It also carries the Plancherel measure $\nu^{P}$, which is characterized by the inversion

1. We will sometimes also use $k$ as notation for certain integers, but no confusion should arise. 
formula

$$
f(1)=\int_{\mathcal{A}_{v}} \operatorname{Tr}(\pi(\tilde{f})) d \nu^{P}(\pi), \quad \tilde{f}(g)=f\left(g^{-1}\right),
$$

for "reasonable" functions $f: \mathbf{G}\left(k_{v}\right) \longrightarrow \mathbf{C}$ (such, in particular, that the trace of $\pi(\tilde{f})$ is well-defined.)

We think of $\mathcal{A}_{v}$ as significantly "simpler" than $\mathcal{A}(\mathbf{G})$, and in particular we expect it to be amenable to many techniques of harmonic analysis.

6. The local spectrum $\mathcal{A}_{v}$ contains some distinguished subsets: the unramified part $\mathcal{A}_{v}^{n r}$ of the spectrum, and the tempered part $\mathcal{A}_{v}^{t}$. The intersection $\mathcal{A}_{v}^{n r} \cap \mathcal{A}_{v}^{t}$, the tempered, unramified, spectrum, is denoted $\mathcal{A}_{v}^{0}$. As in $4, \mathcal{A}_{v}^{n r}$ can be identified naturally with the set of semisimple conjugacy classes in ${ }^{L} \mathbf{G}$, and $\mathcal{A}_{v}^{0}$ can be identified with the set of conjugacy classes in a maximal compact subgroup $K$ of the $L$-group of $\mathbf{G}$.

7. If $S$ is a finite set of places of $k$, we write

$$
\mathcal{A}_{S}=\prod_{v \in S} \mathcal{A}_{v}
$$

considered as given with the product topology, the product Plancherel measure, etc.

8. If $\pi$ is a unitary irreducible representation of $\mathrm{GL}_{n}\left(k_{v}\right)$, we denote by $\mathfrak{q}(\pi)$ its analytic conductor, defined as the usual arithmetic conductor when $v$ is finite, and given by

$$
\mathfrak{q}(\pi)=\prod_{j=1}^{[k: \mathbf{Q}]}\left(1+\left|t_{j}\right|\right)
$$

when $v \mid \infty$, where the $t_{j}$ are the Langlands parameters of $\pi$.

For a cusp form $\pi \in \mathcal{A}\left(\mathrm{GL}_{n}\right)$, we use the analytic conductor as defined by Iwaniec and Sarnak:

$$
\mathfrak{q}(\pi)=\prod_{v} \mathfrak{q}\left(\pi_{v}\right)=q(\pi) \prod_{j=1}^{n[k: \mathbf{Q}]}\left(1+\left|t_{j}\right|\right)
$$

where $q(\pi)$ is the "finite" part of the conductor (the factor appearing in the functional equation of the standard $L$-function of $\pi$ ). This is therefore a positive real number that measures the complexity of $\pi$ both at the finite places (taking into account the ramification) and infinite places.

9. If $\mathbf{G}$ is not $\mathrm{GL}_{n}$, we will always work in a context where there is given a fixed representation $\varrho:{ }^{L} \mathbf{G} \longrightarrow \mathrm{GL}_{n}(\mathbf{C})$ of the $L$-group, with finite kernel, and some associated maps

$$
\varrho_{*, v}: \mathcal{A}_{v}(\mathbf{G}) \longrightarrow \mathcal{A}_{v}\left(\mathrm{GL}_{n}\right)
$$

at all places. For $\pi \in \mathcal{A}(\mathbf{G})$, this allows us to define a corresponding analytic conductor (depending on $\varrho$ )

$$
\tilde{\mathfrak{q}}(\pi)=\prod_{v} \mathfrak{q}\left(\varrho_{*, v} \pi_{v}\right),
$$

(in fact, we will apply this only in this paper in Section 13 for $\mathbf{G}=\mathrm{Sp}_{4}$ and $\pi$ unramified at all finite places.) 
We expect, of course, that $\varrho_{*, v}$ is given by a form of the local Langlands correspondance. Since this is not known yet, ${ }^{(2)}$ this may be problematic, but often we are restricting to the unramified subset of $\mathcal{A}_{v}$, where $\varrho_{*, v}$ is defined by the matching of Satake parameters, i.e., by the induced map from (semisimple) conjugacy classes of ${ }^{L} \mathbf{G}$ to those of $\mathrm{GL}_{n}$.

10. If $\varphi \in L_{0}^{2}\left(\mathbf{G}(k) \backslash \mathbf{G}\left(\mathbf{A}_{k}\right)\right)$ is any automorphic function which generates an automorphic representation $\pi$, or even a $\pi$-isotypic representation $\pi^{\oplus k}$, we will denote $\pi(\varphi)=\pi$, and will also write $\pi_{v}(\varphi)$ for $\pi_{v}$ and $\tilde{\mathfrak{q}}(\varphi)$ for $\tilde{\mathfrak{q}}(\pi)$.

11. Although it seems most natural to study average values of $L$-functions performed using the counting measure (possibly normalized), many different contexts suggest, or make use of, more general weighted sums (including in principle integral averages, typically on intervals of the critical line, as in the "family" $\zeta(1 / 2+i t))$. To handle very general circumstances, we will define a small family of cusp forms on $\mathbf{G} / k$ to be any finite measure $\mu$ on the set $\mathcal{A}(\mathbf{G})$ (with respect to the obvious $\sigma$-algebra; typically, $\mu$ will be supported on a countable set so measurability issues will be non-existent; continuous parameters arise usually only from the critical line, as mentioned above, and then the measurability questions are also clear). We assume the non-degeneracy condition $\mu(\mathcal{A}(\mathbf{G})) \neq 0$. The intuition is that $\mu$ counts, in some maybe "smoothed" way, the cusp forms where certain parameters lie in suitable ranges. In particular, $\mu$ is of highly arithmetic nature, and should be subject to the typical mix of "structure and randomness" one sees in objects of multiplicative number theory and arithmetic geometry.

12. Typical examples are:

- If $\mathcal{F} \subset \mathcal{A}(\mathbf{G})$ is a finite set, the counting measure on it, or the normalized counting measure, where $\mu$ is a probability measure and every element of $\mathcal{F}$ has equal weight; of particular importance are the counting measures associated to

$$
\mathcal{F}_{X}=\{\pi \in \mathcal{A}(\mathbf{G}) \mid \mathfrak{q}(\pi) \leqslant X\},
$$

for $X>0$ (such that the set is non-empty; its finiteness is - or should be - a basic height-like property of the analytic conductor).

- If $w$ is any non-negative function on $\mathcal{A}(\mathbf{G})$ which can be summed (in particular, which is supported on a countable set), one can define a measure $\mu$ with $\mu(\{\pi\})=w(\pi)$. This is often done for families of Maass forms, for instance.

13. Given a small family $\mu$, it is most often the relative average of invariants of cusp forms, measured with respect to $\mu$, which is important. For instance, when dealing with the family $\mathcal{F}_{X}$, the asymptotic behavior of $\left|\mathcal{F}_{X}\right|$ is by no means easy to fathom, but one may expect that many results are best interpreted as dealing with

$$
\frac{1}{\left|\mathcal{F}_{X}\right|} \sum_{\pi \in \mathcal{F}_{X}} \alpha(\pi)
$$

Thus, in general, we will write

$$
\mathbf{E}_{\mu}(\alpha)=\mathbf{E}_{\mu}(\alpha(\pi))=\frac{1}{\mu(\mathcal{A}(\mathbf{G}))} \int_{\mathcal{A}(\mathbf{G})} \alpha(\pi) d \mu(\pi),
$$

2. It is known for $\mathrm{GL}_{n}$ by the work of Harris and Taylor, and for $\mathrm{GSp}_{4}$, by work by Gan and Takeda. 
for any (measurable, if need be) function $\alpha$ defined on $\mathcal{A}(\mathbf{G})$, and also introduce the corresponding (redundant-looking) probability notation:

$$
\mathbf{P}_{\mu}(\{\pi \text { satisfies } \ldots\})=\frac{1}{\mu(\mathcal{A}(\mathbf{G}))} \mu(\{\pi \in \mathcal{A}(\mathbf{G}) \mid \pi \text { satisfies } \ldots\}) .
$$

For $\mathcal{F}_{X}$ (and similarly later for other families defined using some parameter similar to the "height bound" $X$ that appears here, e.g. $\left.\mu_{n}\right)$, we will use the shorthand notation $\mathbf{E}_{X}(\cdots)$ and $\mathbf{P}_{X}(\cdots)$ (resp. $\mathbf{E}_{n}(\cdots)$, etc).

14. For a small family $\mu$ of cusp forms, we denote by $\mu_{v}$ the image of $\mu$ under the map $\pi \mapsto \pi_{v}$ on $\mathcal{A}(\mathbf{G})$ (which should have all necessary measurability properties to make $\mu_{v}$ a Borel measure on $\mathcal{A}(\mathbf{G})$ ). Similarly, for a finite set $S$ of places, we write $\mu_{S}$ for the image measure of $\mu$ in $\mathcal{A}_{S}$. In other words, for nice subsets $B_{v} \subset \mathcal{A}_{v}$, defined for $v \in S$, we have

$$
\mu_{S}(B)=\mu\left(\left\{\pi \in \mathcal{A}(\mathbf{G}) \mid \pi_{v} \in B_{v} \text { for } v \in S\right\}\right) .
$$

15. The local equidistribution question for a small family $\mu$ asks, roughly, "what do the local measures $\mu_{v}$ look like?", where it is thought that $v$ is fixed. What is expected is that, under suitable conditions, $\mu_{v}$ is "close" to a nice intrinsically-defined measure on $\mathcal{A}_{v}$. This target measure, say $\nu_{v}$, is in many (but not all) cases rather impervious to the arithmetic subtleties of the small family.

16. Assuming that some form of the local equidistribution question is answered positively, the local independence question asks whether the measure $\nu_{v}$ above, if they are defined for all $v$ in some set $S$ of places, have the property that the image measure $\mu_{S}$ (living on the set of possible $S$-tuples of local components of cusp forms "in" $\mu$ ) is close to the product

$$
\prod_{v \in S} \nu_{v}
$$

(if true, this indicates that there is no obvious restriction on $\pi_{v}$, for some $v \in S$, even when knowing all the other $\left.\pi_{w}, w \in S-\{v\}\right)$.

17. For a more precise formulation, involving a well-defined question, we must assume given a sequence $\left(\mu_{n}\right)$ of small families on $\mathcal{A}(\mathbf{G})$ (the parameter $n$ going to infinity could of course be replaced by any similar one, e.g., one can take the families $\left(\mu_{X}\right)$ of cusp forms with analytic conductor $\leqslant X$, with $X \longrightarrow+\infty$ ), which we think as covering a larger and larger (somehow coherent) set of cusp forms, possibly with some weight. Then we have image measures $\mu_{v, n}$ on $\mathcal{A}_{v}$ for all places $v$ and for all parameters $n$. We will call such a collection an extended family. ${ }^{(3)}$ The basic questions, where the second is stronger than the first (and the third is a variant), are:

1. For a fixed place $v$ of $k$, does there exist a Borel measure $\nu_{v}$, independent of $n$, on $\mathcal{A}_{v}$, such that $\mu_{v, n}$ converges weakly to $\nu_{v}$ as $n \longrightarrow+\infty$ ?

3. This is very provisional terminology; a better one would emphasize the fact that these are not yet at all supposed to be well-behaved families worthy of the name. 
2. Do there exist Borel measures $\nu_{v}$ on $\mathcal{A}_{v}$, independent of $n$, defined for all places $v$, such that for any finite set $S$ of places, the measures $\mu_{S, n}$ converge weakly to

$$
\nu_{S}=\prod_{v \in S} \nu_{v}
$$

as $n \longrightarrow+\infty$ ?

3. If $k$ is a number field: do there exist Borel measures $\nu_{v}$ on $\mathcal{A}_{v}$, independent of $n$, defined for all finite places $v$, such that for any finite set $S$ of finite places, the measures $\mu_{S, n}$ converge weakly to

$$
\nu_{S}=\prod_{v \in S} \nu_{v}
$$

as $n \longrightarrow+\infty$ ?

In probabilistic terms, (2) means that the local components $\pi_{v}$ of the small families $\mu_{n}$ are asymptotically independent as $n \rightarrow+\infty$.

18. In most cases, we expect that if local spectral equidistribution holds, the limiting measure $\nu_{v}$ is supported on the tempered spectrum $\mathcal{A}_{v}^{t}$. When $\mathbf{G}=\mathrm{GL}_{n}$, this should be automatic because of the Ramanujan-Petersson conjecture, but even when there are cusp forms on $\mathbf{G}$ for which the Ramanujan-Petersson conjecture fails (e.g., on $\mathbf{G}=\mathrm{Sp}_{4}$ because of the SaitoKurokawa lifts), these exceptions will typically be few (unless they are specifically targeted in the family!). For an example, see Section 13.

Similarly, the limiting measure $\nu_{v}$ is sometimes supported on the unramified part of the spectrum, but it may also occur quite frequently that the fact that ramification is "rare" is displayed instead in the fact that the mass $\nu_{v}\left(\mathcal{A}_{v}^{n r}\right)$ of the unramified spectrum is "large", and in particular converges to 1 as $N v$ tends to infinity. This plays a big role later in Section 9.

19. Since the analytic conductor has height-like properties, it is necessary that an extended family $\left(\mu_{n}\right)$ "involve" in a significant way more and more cusp forms with increasing conductor, as $n$ grows. To measure this growth, we define

$$
\kappa(n)=\mathbf{E}_{n}(\tilde{\mathfrak{q}}(\pi))
$$

the average conductor for $\mu_{n}$. We will always have $\kappa(n) \rightarrow+\infty$ as $n \rightarrow+\infty$, and we will use this quantity in estimates related to quantitative forms of equidistribution (Section 8).

\section{Families of $L$-functions}

20. The most important invariants of families of cusp forms are related to their $L$-functions. Thus, by (extended) family of L-functions associated to an (extended) family $\left(\mu_{n}\right)$, we mean that we are given a representation

$$
\varrho:{ }^{L} \mathbf{G} \longrightarrow \mathrm{GL}_{m}(\mathbf{C})
$$

of the $L$-group, and we consider the asymptotic behavior of the Langlands $L$-functions $L(\pi, \varrho, s)$ associated to the representations $\pi$ in the family using $\varrho$. Note that we will always mean by $L$-function the finite part of the completed $L$-function, i.e., without Gamma factors. Thus we will consider averages like

$$
\mathbf{E}_{n}\left(L\left(\pi, \varrho, s_{0}\right)\right), \quad \mathbf{E}_{n}\left(L(\pi, \varrho, 1 / 2)^{k}\right), \quad \text { etc }, \ldots
$$


or averages involving the distribution of zeros of $L(\pi, \varrho, s)$.

21. Any $L$-function $L(\pi, \varrho, s)$ has also an analytic conductor, which we denote $\mathfrak{q}\left(\varrho_{*} \pi\right)$ (with a slight abuse of notation); it coincides with the conductor $\tilde{\mathfrak{q}}(\boldsymbol{\pi})$ defined in $\mathbf{9}$, computed using $\varrho$.

22. When $\mathbf{G}=\mathrm{GL}_{d}$ (unless specified otherwise) the family of $L$-functions is the family of standard $L$-functions associated to the tautological representation ${ }^{L} \mathrm{GL}_{d}=\mathrm{GL}_{d}(\mathbf{C}) \stackrel{=}{\longrightarrow}$ $\mathrm{GL}_{d}(\mathbf{C})$. Then $\tilde{\mathfrak{q}}(\pi)=\mathfrak{q}(\pi)$ is the "standard" analytic conductor (see 8). However, keeping track of a general representation $\varrho$ will lead to some clarifications, for instance when it comes to the determination of the "symmetry type" of an extended family of $L$-functions.

23. For a general group $\mathbf{G}$ with representation $\varrho$ as above, we recall that the $L$-function $L(\pi, \varrho, s)$ is defined by an Euler product

$$
L(\pi, \varrho, s)=\prod_{v \text { finite }} L_{v}\left(\pi_{v}, \varrho, s\right),
$$

where $L_{v}\left(\pi_{v}, \varrho, s\right)$ is a local $L$-function which is meant to be defined in general using a local Langlands correspondance

$$
\varrho_{*, v}: \mathcal{A}_{v}(\mathbf{G}) \longrightarrow \mathcal{A}_{v}\left(\mathrm{GL}_{n}\right)
$$

(which is just the identity if $\mathbf{G}=\mathrm{GL}_{n}$ and $\varrho$ is the identity, defining the standard $L$-function), i.e., we expect to be able to define

$$
L_{v}(\theta, \varrho, s)=L_{v}\left(\varrho_{*, v} \theta, s\right)
$$

for all places $v$ and all local representations $\theta \in \mathcal{A}_{v}(\mathbf{G})$ (we use the notation $\theta$ to emphasize that these representations are arbitrary, and not necessarily related to global representations.) These local correspondances are not known to be defined in all cases, with the expected properties. However, for an unramified representation $\theta \in \mathcal{A}_{v}^{n r}(\mathbf{G})$, the local map is always defined, as recalled in $\mathbf{9}$, and the local $L$-factor is given by

$$
L_{v}(\theta, \varrho, s)=L_{v}\left(\varrho_{*, v} \theta, s\right)=\operatorname{det}\left(1-(N v)^{-s} \varrho(\Theta)\right)^{-1},
$$

for $\theta \in \mathcal{A}_{v}(\mathbf{G})$. in terms of the semisimple conjugacy class $\Theta$ in ${ }^{L} \mathbf{G}$ parameterizing the unramified representation $\theta$.

Thus $L(\pi, \varrho, s)$ is always well-defined, using the Euler product, if $\pi$ is everywhere unramified at the finite places.

24. It is part of the Langlands conjectures that, in fact, there exists a global functoriality map

$$
\varrho_{*}: \mathcal{A}(\mathbf{G}) \longrightarrow \mathcal{A}\left(\mathrm{GL}_{n}\right)
$$

(possibly defined on a subset of $\mathcal{A}(\mathbf{G})$ to ensure that the image is cuspidal, as one sees even for the symmetric square on $\mathrm{GL}_{2}$ ), with the property, among others, that

$$
L\left(\varrho_{*} \pi, s\right)=L(\pi, \varrho, s),
$$

or in other words, the Euler product (2) is in fact a standard $L$-function of a cusp form on $\mathrm{GL}_{n}$.

25. However, statements of Langlands functoriality in this sense are still rather rare when $k$ is a number field. Thus it is indeed useful to be able to work efficiently with the $L$-function $L(\pi, \varrho, s)$ as an invariant of $\pi$. This can be done in quite a few cases, with local factors 
for $L(\pi, \varrho, s)$ well-defined at all places (including ramified places), and the "usual" analytic properties - functional equation, polynomial growth in vertical strips, as described in [10, Ch. 5]. Even if only partial analytic continuation, or meromorphic continuation with partial location of the poles is known, one can still consider the values of $L(\pi, \varrho, s)$ where it does make sense.

For instance, one can consider general Rankin-Selberg convolutions of cusp forms on $\mathrm{GL}_{m_{1}}$ by cusp forms on $\mathrm{GL}_{m_{2}}$, since the analytic properties of the $L$-functions are known (due to the work in particular of Mœglin-Waldspurger, and Jacquet-Piatetski Shapiro-Shalika).

In the unramified case (at finite places), at least in the region of absolute convergence, one can consider arbitrary $\varrho$, using the definition (3), since it is known from "trivial" bounds on Satake parameters that the Euler product converges in some rightward half-plane.

\section{Analogy with sieve}

26. This section may certainly be omitted in a first reading. We explain how some of the basic ideas above are analogues of the general sieve settings described in [13].

27. In that framework, one is given a set $Y$ of "global" objects of interest, and one wishes to extract information related to "sifted sets". These are defined using local information maps

$$
\varrho_{\ell}: Y \longrightarrow Y_{\ell}
$$

with $Y_{\ell}$ finite, where $\ell$ runs through some (general) index set $\Lambda$, and are of the form

$$
\mathcal{S}=\left\{y \in Y \mid \varrho_{\ell}(y) \notin \Omega_{\ell} \text { for all } \ell \in \mathcal{L}\right\}
$$

for certain subset $\mathcal{L}$ of indices and certain sifting conditions $\Omega_{\ell} \subset Y_{\ell}$.

Specifically, one is given a finite (often probability) measure $\mu$ on $Y$, or a sequence $\left(\mu_{n}\right)$ of such measures, and one wishes to compute $\mu(\mathcal{S})$.

28. As an example, take $Y=\mathrm{SL}_{n}(\mathbf{Z})$, and let $\varrho_{\ell}$, for $\ell$ prime, be the reduction map modulo $\ell$, which surjects to $Y_{\ell}=\mathrm{SL}_{n}\left(\mathbf{F}_{\ell}\right)$. Define $\Omega_{\ell}$ to be the set of matrices in $Y_{\ell}$ with irreducible characteristic polynomial. Then, for any $\mathcal{L}, \mathcal{S}$ contains the $g \in \mathrm{SL}_{n}(\mathbf{Z})$ for which $\operatorname{det}(T-g)$ is reducible.

29. As explained in [13], sieve methods apply with some success provided the following two conditions are satisfied: (1) for each $\ell$, the measure $\varrho_{\ell, *} \mu$ is "close" to some measure $\nu_{\ell}$ on $Y_{\ell}$ - typically, $\varrho_{\ell, *} \mu_{n}$ converges to $\nu_{\ell}$ as $n$ grows; (2) for a finite subset $S$ of the index set of $\ell$ 's, the measures

$$
\prod_{\ell \in S} \varrho_{\ell, *} \mu
$$

(on the product of $Y_{\ell}$ over $\ell \in S$ ) are close, or converge to, the product measure of the $\nu_{\ell}$.

30. Except for the fact that families of cusp forms (seen as global objects) involve as local information the local components $\pi_{v}$, which live in infinite sets $\mathcal{A}_{v}$, we see that the formal setting and the basic local equidistribution questions of Section 1 are exactly of this type. One can indeed perform a certain amount of sieving (especially of the "large sieve" type) for extended families of cusp forms with local equidistribution, especially when a quantitative form holds (see Section 8). 


\section{Examples of local equidistribution}

31. We give some easy examples now, involving $k=\mathbf{Q}$ and $\mathbf{G}=\mathrm{GL}_{1}$ or $\mathbf{G}=\mathrm{GL}_{2}$. The small families involved are the following (where we simply write down the finite support of a measure when the normalized (probability) counting measure is meant, and where we write primitive Dirichlet characters and classical, holomorphic and Maass, primitive cusp forms, with respect to some Hecke group $\Gamma_{0}(q)$, to denote the corresponding cuspidal automorphic representations of $\mathrm{GL}_{2}$ over $\left.\mathbf{Q}\right)$ :

1. $D_{q}$ is the set of all primitive Dirichlet characters of conductor $q$;

2. $D_{Q}^{+}$is the set of all primitive Dirichlet characters of conductor $\leqslant Q$;

3. $C_{k}$ is the set of all primitive holomorphic cusp forms of weight $k$ and level 1 ;

4. $C_{k, q}$ is the set of all primitive holomorphic cusp forms of weight ${ }^{(4)} k$ and level $q ;^{(5)}$

5. $C_{k, Q}^{+}$is the set of all primitive holomorphic cusp forms of weight $k$ and level $\leqslant Q$;

6. $C_{K, q}^{++}$is the set of all primitive holomorphic cusp forms of weight $\leqslant K$ and level $q$;

7. $C_{K, Q}^{++}$is the set of all primitive holomorphic cusp forms of weight $\leqslant K$ and level $\leqslant Q$;

8. For any of the $C$-sets above, say $C_{\star}^{\square}$, the small family $H_{\star}^{\square}$ has the same support but the measure $\mu=\mu_{\star}^{\square}$ is obtained by putting on a newform $f$ the weight

$$
\omega(f)=\frac{\Gamma(k-1)}{(4 \pi)^{k-1}} \frac{1}{\langle f, f\rangle}
$$

where $\langle\cdot, \cdot\rangle$ is the Petersson inner product. This is the "harmonic weight" that arises from the Petersson formula.

9. More variants involving the choice of a non-trivial nebentypus can also be considered...

10. Even more variants can be constructed with Maass forms; let us define only $M_{T}$ and $H_{T}$, where $M_{T}$ is the set of primitive Maass forms of level 1 and Laplace eigenvalue $1 / 4+t^{2}, 0 \leqslant t \leqslant T$, and where $H_{T}$ is the corresponding measure with weight given by the analogue of the Petersson weight above.

32. For $\mathrm{GL}_{1}$ (and the extended families $\left(D_{q}\right)$ and $\left(D_{Q}^{+}\right)$, with asymptotic parameters $q \rightarrow+\infty$, possibly along a subsequence of integers, or $Q \rightarrow+\infty$ respectively, the local equidistribution problem (which we consider only for the finite places) has not been considered previously in the literature - to the author's knowledge -, but it is extremely simple and elementary. Here is a quick summary.

33. The basic information needed is, for a given $p$, the description of the local character of $\mathbf{Q}_{p}^{\times}$associated with a primitive Dirichlet character $\chi$. Once we select the prime $p$ itself as a uniformizer at $p$, we obtain an isomorphism of the dual of $\mathbf{Q}_{p}^{\times}$with $\mathbf{Z}_{p}^{\times} \times \mathbf{S}^{1}$, where the factor $\mathbf{S}^{1}$ corresponds to unramified characters. The component $\chi_{p}$ of $p$ is given as follows: factor ${ }^{(6)}$

\footnotetext{
4. In this section, the weight is always at least 2 .

5. Unless specified otherwise, a classical cusp form of level $q$ is considered to have trivial nebentypus.

6. Here we use crucially the fact, specific to $\mathrm{GL}_{1}$ or abelian groups more generally, that the dual of $\mathbf{Q}_{p}^{\times}$is still a group.
} 
$\chi=\chi_{p} \chi_{p}^{\prime}$, where $\chi_{p}$ is a character of conductor a power of $p$, say $p^{k}, k \geqslant 0$, and $\chi_{p}^{\prime}$ is one with conductor coprime to $p$; then

$$
\chi_{p}=\left(\tilde{\chi}_{p}, \chi_{p}^{\prime}(p)\right) \in \mathbf{Z}_{p}^{\times} \times \mathbf{S}^{1},
$$

with the ramified component $\tilde{\chi}_{p}$ obtained by the inverse of the composite

$$
\mathbf{Z}_{p}^{\times} \longrightarrow\left(\mathbf{Z} / p^{k} \mathbf{Z}\right)^{\times} \stackrel{\chi_{p}}{\longrightarrow} \mathbf{S}^{1}
$$

In particular, if $\chi$ is unramified at $p$, the local component can be (canonically here) identified with the value $\chi(p) \in \mathbf{S}^{1}$.

34. Consider first $D_{q}$, i.e., primitive characters with a given level. If all $q$ are considered, this does not satisfy local equidistribution, simply because for any fixed prime $p$, there is, if $p \mid q$, a non-zero probability (depending only on $p$ ) that $\chi \in D_{q}$ be ramified, while this probability is zero for $p \nmid q$. Thus the $\mu_{q}$-measure of the unramified dual of $\mathbf{Q}_{p}$ does not have a limit as $q \rightarrow+\infty$.

35. However, if we restrict $q$ to any sequence where the smallest prime divisor $P^{-}(q)$ tends to infinity, there is strong local equidistribution in $\left(D_{q}\right)$ thus restricted. The reason is that, for any finite set of primes $S$, all $\chi \in D_{q}$ are unramified at all $p \in S$ when $q$ is large enough. It is then an easy matter to check that $\chi_{p}$, for $p \in S$, becomes equidistributed according to the probability Haar measure on the unramified dual of $\mathbf{Q}_{p}$ (isomorphic to $\mathbf{S}^{1}$ ), and that the $S$-components become equidistributed with respect to the product of these measures.

36. It is also interesting to look at the extended family $\left(D_{Q}^{+}\right)$of primitive characters of modulus $\leqslant Q$. Then, as $Q \rightarrow+\infty$ (without restriction), there is strong local equidistribution, with limiting measures $\nu_{p}$ on the dual of $\mathbf{Q}_{p}$ that can be described as follows: on each connected component $\left\{\chi_{0}\right\} \times \mathbf{S}^{1}$, the unramified component is "relatively" equidistributed with respect to the Haar measure on $\mathbf{S}^{1}$, while the connected component has total measure equal to

$$
\nu_{p}\left(\left\{\chi_{0}\right\} \times \mathbf{S}^{1}\right)=\frac{p^{3}}{(p-1)(p+1)^{2}} \frac{1}{p^{2 k}}
$$

where $k$ is the exponent of the conductor of the local character $\chi_{0}$. This is again a fairly simple computation (one "sees" $\chi_{0}$ as a primitive character modulo $p^{k}$, and notes that the characters $\chi$ modulo $q \leqslant Q$ which have $\chi_{p}$ equivalent to $\chi_{0}$, modulo an unramified character, exist only when $q=p^{k} r$ with $p \nmid r$, and are then exactly those of the form $\chi_{0} \chi^{\prime}$ where $\chi^{\prime}$ is primitive modulo $r$.) A first useful check of the formula above is the fact that it does correspond to a probability measure on the dual of $\mathbf{Q}_{p}$; this follows from

$$
\frac{p^{3}}{(p-1)(p+1)^{2}} \sum_{k \geqslant 0} \frac{1}{p^{2 k}} \sum_{\chi_{0}\left(\bmod p^{k}\right)}^{*} 1=1,
$$

(where the inner sum is the number of primitive character modulo $p^{k}$ ), which is correct, and is a rather simple computation.

This measure on the dual of $\mathbf{Q}_{p}$ is somewhat unusual. It is not the Plancherel measure (which, being a Haar measure on the dual group, treats each connected component as equal!), though they are mutually absolutely continuous. Note - for further reference - that its mystery evaporates as $p \rightarrow+\infty$ : $\nu_{p}$ "converges" then to the probability Haar measure on the unramified spectrum. (We put quotes here because, in principle, the measures $\nu_{p}$ live on 
different spaces for $p$ varying, where however the unramified component can be all identified; one should then say that the restriction of $\nu_{p}$ to the unramified component, after identification to the "constant" space $\mathbf{S}^{1}$, converges to probability Haar measure on $\mathbf{S}^{1}$.) We will see later that this type of "large $p$ limit" is very frequent and has important consequences, e.g., with respect to the determination of the conjectural symmetry type of the family (see Section 9).

37. For classical cusp forms, local equidistribution at unramified places was proved by Serre [22]. Precisely, consider a sequence of pairs $(k, q)$, where $k$ is a weight and $q$ a level, such that $k+q \rightarrow+\infty$, and a finite set of primes $S$ such that no prime in $S$ divides infinitely many $q$ 's. Then, for $q$ large enough, any $f \in C_{k, q}$ is unramified at all $p \in S$. The local component at $p$ of the cusp form $\pi(f)$ associated to $f$ is very easily described in terms of its Satake parameters $\left(\alpha_{p}, \beta_{p}\right)$, namely we have

$$
\alpha_{p}+\beta_{p}=\lambda_{f}(p)
$$

the Hecke eigenvalue at $p$, and $\alpha_{p} \beta_{p}=1$ (this last is because we work without nebentypus...). In fact there is then an identification of the space of possible local components (the part of the spectrum of $\mathrm{GL}_{2}\left(\mathbf{Q}_{p}\right)$ corresponding to unramified, tempered ${ }^{(7)}$ representations with trivial central character) with the interval $[-2,2]$, in which $\pi_{p}(f)$ corresponds to $\lambda_{f}(p)$ for $f$ unramified at $p$. Serre shows local equidistribution of $\pi_{S}(f)$ for $f \in C_{k, q}$, with respect to the product measures $\nu_{S}=\prod \nu_{p}$, where $\nu_{p}$ is the Plancherel measure on the (unramified, tempered, trivial central character) spectrum of $\mathrm{GL}_{2}\left(\mathbf{Q}_{p}\right)$. This measure $\nu_{p}$ is given explicitly by

$$
\nu_{p}=\frac{p+1}{\left(p^{1 / 2}+p^{-1 / 2}\right)^{2}-x^{2}} \frac{1}{\pi} \sqrt{1-\frac{x^{2}}{4}} d x .
$$

In particular, this proves strong local equidistribution in level 1, with Plancherel measures as limiting measures, for the family $C_{k, 1}$. Royer [19] has also proved such results quantitatively.

38. If one uses instead the families $H_{k, q}$ (with harmonic weight on $C_{k, q}$ ), it follows almost immediately from the Petersson formula that (at least in the absence of oldforms) there is strong local equidistribution of the $p$-components for $p \in S$, again for levels coprime to $S$, but with limiting measure $\tilde{\nu}_{p}$ at $p$ equal to the Sato-Tate measure (which is independent of $p$ ) on the unramified tempered (trivial central character) spectrum, identified with $[-2,2]$ as above:

$$
\tilde{\nu}_{p}=\frac{1}{\pi} \sqrt{1-\frac{x^{2}}{4}} d x .
$$

39. Similarly, Sarnak obtained local equidistribution for Maass forms [21] (at unramified places); the weighted version goes back (implicitly) to Bruggeman's work.

40. The most general result currently available (to the author's knowledge) is due to SugWoo Shin [23], who proves local equidistribution with the Plancherel measure as limiting measures for families of cusp forms on groups with discrete series as components at infinity (this excludes $\mathrm{GL}_{n}, n \geqslant 3$, but includes $\mathrm{GSp}_{2 g}$ ). We will say a bit more in Section 8, when discussing the recent quantitative refinements announced by Shin and Templier [24].

7. Because of Deligne's bound $\left|\alpha_{p}\right|=\left|\beta_{p}\right|=1$. 


\section{Families of cusp forms according to Sarnak's letter}

41. In the letter [20], P. Sarnak gives a list of certain "canonical" types of families of cusp forms on $\mathrm{GL}_{n}$ over $\mathbf{Q}$; these are all defined by taking a set $\mathcal{F}$ of cusp forms in $\mathcal{A}\left(\mathrm{GL}_{n}\right)$ (which is usually infinite) of special type, and then considering - in a different language - the extended family $\left(\mathcal{F}_{X}\right)$ with normalized counting measure on

$$
\mathcal{F}_{X}=\{\pi \in \mathcal{F} \mid \mathfrak{q}(\pi) \leqslant X\} .
$$

42. The special types of sets $\mathcal{F}$ considered by Sarnak encompass essentially all types of arithmetic restrictions on concrete incarnations of families that have arisen "in practice". These are the following, ${ }^{(8)}$ listed with the shorthand names attached to them by Sarnak:

1. [Degenerate case] $\mathcal{F}$ is a single cusp form $\pi$;

2. [Root numbers and central characters] $\mathcal{F}$ is either the set of $\pi$ with a fixed central character, or the set of self-dual forms in $\mathcal{A}\left(\mathrm{GL}_{n}\right)$, or the set of self-dual forms with a fixed sign \pm 1 of the functional equation.

3. [Given $H$-type] ${ }^{(9)}$ Here $H \subset \mathrm{GL}_{n}(\mathbf{C})$ is a semisimple subgroup and $\mathcal{F}$ (which depends on $H$ ) is the set of all cusp forms $\pi$ such that the semisimple conjugacy classes in $\mathrm{GL}_{n}(\mathbf{C})$ associated to the unramified components of $\pi$ intersect $H$ (for almost all places).

4. [Harmonic analysis constraints] Here one fixes a finite set of places $S$ and "nice" subsets $B_{v}$ of the local tempered spectrum of $\mathrm{GL}_{n}\left(\mathbf{Q}_{v}\right)$ for $v \in S$, and $\mathcal{F}$ might be either the set of cusp forms unramified outside $S$, but with local components in $B_{v}$ for $v \in S$; or simply the set of cusp forms with $\pi_{v} \in B_{v}$ for $v \in S$, but possibly still ramified outside $S$.

5. [Geometric families] Here one takes a nice family of algebraic varieties $V \longrightarrow T$, and one picks a family $H_{t}$ of (fixed index) cohomology groups of $V \longrightarrow T, t \in T$; these are conjecturally - and very special cases are known - associated to cusp forms $\pi(t)$ on $\mathrm{GL}_{n}$ with $n$ the constant dimension of $H_{t}$, and $\mathcal{F}$ is the set of these $\pi(t)$. ${ }^{(10)}$

6. [Functorial transfers] Given a reductive group $\mathbf{H} / \mathbf{Q}$ with a representation $\varrho:{ }^{L} \mathbf{H} \longrightarrow$ $\mathrm{GL}_{n}(\mathbf{C})$, and the associated (usually conjectural) Langlands functoriality map $\varrho_{*}$ : $\mathcal{A}(\mathbf{H}) \longrightarrow \mathcal{A}\left(\mathrm{GL}_{n}\right)$, let $\mathcal{F}$ be the image of $\varrho_{*}{ }^{(11)}$

43. Sarnak further defines families of L-functions as follows. One takes finitely many families $\mathcal{F}_{i}$ of the type above (for $\mathrm{GL}_{n_{i}}, 1 \leqslant i \leqslant k$ ) and the product family

$$
\mathcal{F}=\prod_{i} \mathcal{F}_{i},
$$

and then one considers representations $\varrho_{i}: \mathrm{GL}_{n_{i}}(\mathbf{C}) \longrightarrow \mathrm{GL}_{m_{i}}(\mathbf{C})$, and their associated Langlands transfers $\varrho_{i, *}$ - of course usually conjectural! -, in order to obtain an extended

8. Some, for instance (2), could be split further, but we keep the terminology in Sarnak's text.

9. One should think of this as "generalized CM" cases.

10. Here one should really take the possible multiplicity of the $\pi(t)$ 's into account, as managing it analytically is usually pretty much out of the question. This is one of the motivations for using a more general way of "counting" in the definition of small families.

11. Here also multiplicity should probably be taken into account. 
family with

$$
\mathcal{F}_{X}=\left\{\pi=\bigotimes_{i} \pi_{i} \in \mathcal{F} \mid \mathfrak{q}\left(\bigotimes_{i} \pi_{i}\right) \leqslant X\right\}
$$

(the tensor product of the $\pi_{i}$ 's is supposed to be the generalized Rankin-Selberg product, a cusp form on $\mathrm{GL}_{n_{1} \cdots n_{k}}$, which is conjectured to exist).

Then one wants to study particularly the properties of the $L$-functions

$$
L\left(\bigotimes_{i} \varrho_{i, *}\left(\pi_{i}\right), s\right)
$$

using the average over $\mathcal{F}_{X}$.

44. The Sarnak extended families $\mathcal{F}_{X}$ are well-defined (possibly with restrictions related to functoriality in some cases). The following is therefore an unambiguous mathematical questions, which seems fundamental:

For $\mathcal{F}_{X}$ any family as above, does it satisfy strong local equidistribution at the finite places?

It seems very likely that the answer is yes. It would be interesting to know the corresponding limiting measures $\nu_{v}$ (for some of the types at least). The case of $\mathrm{GL}_{1}$ and $k=\mathbf{Q}$ is the content of 36. In any case, one can see that the answer can not be the Plancherel measure, because the latter has infinite total measure. Intuitively, the difference occurs because families of global cusp forms emphasize the unramified part of the spectrum of the local components, which the Plancherel measure does not do to such an extent.

\section{Families of $L$-functions according to Conrey, Farmer, Keating, Rubinstein and Snaith}

45. Another "working definition" of family has been used by Conrey, Farmer, Keating, Rubinstein and Snaith in their paper [6] suggesting a heuristic method to estimate (with lower order terms) moments of $L$-functions.

46. The main difference between [6] and Sarnak's letter (and the point of view taken here) is the focus by the five authors on families of Dirichlet series, without assuming the presence of underlying cusp forms. So they start with a set $\mathcal{F}$ of series

$$
L(s)=\sum_{n \geqslant 1} f(n) n^{-s},
$$

assumed to satisfy Euler product expansions (of some fixed degree $d$ ) and standard functional equations. These are ordered with respect to the log-conductor, which is essentially the same as the logarithm of the analytic conductor if $L(s)$ is a standard $L$-function on $\mathrm{GL}_{d}$; one denotes $\mathcal{F}(T)$ the set of series with $\mathfrak{q}(L(s))<T$. A set of such series is then a cfkrs-family when, ${ }^{(12)}$

12. Conrey, Farmer, Keating, Rubinstein and Snaith impose extra conditions, e.g., of monotonicity of parameters with respect to the conductor, that we ignore here. 
for every $k \geqslant 1$, for every integer $0 \leqslant \ell \leqslant k$, every $k$-tuple of positive integers $\boldsymbol{m}=\left(m_{i}\right)$, the limits

$$
\delta_{\ell}(\boldsymbol{m})=\lim _{T \rightarrow+\infty} \frac{1}{|\mathcal{F}(T)|} \sum_{f \in \mathcal{F}(T)} f\left(m_{1}\right) \cdots f\left(m_{\ell}\right) \overline{f\left(m_{\ell+1}\right) \cdots f\left(m_{k}\right)}
$$

exist, and are multiplicative functions, in the sense that

$$
\delta_{\ell}(\boldsymbol{n m})=\delta_{\ell}(\boldsymbol{n}) \delta_{\ell}(\boldsymbol{m})
$$

for each $\ell$, and tuples $\boldsymbol{n}, \boldsymbol{m}$ with $\left(n_{1} \cdots n_{k}, m_{1} \cdots m_{k}\right)=1$ (the product of tuples on the left of this condition is taken coordinate-wise).

47. Assume we are given an extended family $\mathcal{F}=\left(\mathcal{F}_{n}\right)_{n}$ from finite sets of cusp forms on $\mathrm{GL}_{d}$ over Q, satisfying strong local equidistribution (in our sense) at the finite places, with limit measures $\nu_{p}$. Consider the associated standard $L$-functions $L(\pi, s)$ (restricting to the finite places) for $\pi \in \mathcal{F}$, and expand them

$$
L(\pi, s)=\sum_{n \geqslant 1} \lambda_{\pi}(n) n^{-s}
$$

Then the corresponding limits (7) exist, and satisfy the multiplicativity property (8). In other words, we obtain a cfkrs-family automatically.

To see this, note that for each prime $p$, each integer $\nu \geqslant 0$, the coefficient

$$
\lambda_{\pi}\left(p^{\nu}\right)
$$

is the value at $\pi$ of a continuous function $t_{p, \nu}$ defined on $\mathcal{A}_{p}\left(\mathrm{GL}_{d}\right)$ (in the unramified case, it is a symmetric polynomial in the Satake parameters). Therefore, in terms of the factorization of the $m_{i}$ S's, we have

$$
\lambda_{\pi}\left(m_{1}\right) \cdots \lambda_{\pi}\left(m_{\ell}\right) \overline{\lambda_{\pi}\left(m_{\ell+1}\right) \cdots \lambda_{\pi}\left(m_{k}\right)}=\prod_{p} \prod_{1 \leqslant i \leqslant \ell} t_{p, v_{p}\left(m_{i}\right)}(\pi) \prod_{\ell+1 \leqslant i \leqslant k} \overline{t_{p, v_{p}\left(m_{i}\right)}(\pi)} .
$$

For a fixed $k$-tuple $\boldsymbol{m}$, the product ranges only over a finite set of primes $S$. Thus, by definition, strong equidistribution for $S$ yields the value

$$
\prod_{p \in S} \prod_{1 \leqslant i \leqslant \ell} \int_{\mathcal{A}_{p}} t_{p, v_{p}\left(m_{i}\right)}(\theta) d \nu_{p}(\theta) \prod_{\ell+1 \leqslant i \leqslant k} \overline{\int_{\mathcal{A}_{p}} t_{p, v_{p}\left(m_{i}\right)}(\theta)} d \nu_{p}(\theta)
$$

of the limit as $n \rightarrow+\infty$. This formula is clearly multiplicative in the sense (8).

\section{Direct consequences of strong local equidistribution}

48. Strong local equidistribution, when it holds, has by itself a number of interesting consequences. However, the deepest ones depend on quantitative forms of local equidistribution, to be discussed in Section 8.

49. We assume here that we are given an extended family $\left(\mu_{n}\right)$ on $\mathcal{A}(\mathbf{G})$ which satisfies strong local equidistribution at the finite places. We denote by $\nu_{v}$ the corresponding limit measure on $\mathcal{A}_{v}=\mathcal{A}_{v}(\mathbf{G})$ at the place $v$. 
50. The basic idea is that if $\pi \mapsto \alpha(\pi)$ is a multiplicative invariant, i.e., one that can be expressed as a product over finite places of local quantities $\alpha_{v}\left(\pi_{v}\right)$, then we expect that

$$
\mathbf{E}_{n}(\alpha(\pi)) \longrightarrow \prod_{v \text { finite }} \int_{\mathcal{A}_{v}} \alpha_{v}(\theta) d \nu_{v}(\theta)
$$

as $n \longrightarrow+\infty$.

When valid, this expresses some form of independence of the local components at the finite places, and probabilistically corresponds to the fact that the expectation of a product of independent random variables is the product of the individual expectations.

By definition of strong local equidistribution, (9) holds when each $\alpha_{v}$ is a bounded continuous function on $\mathcal{A}_{v}$, and $\alpha_{v}=1$ for $v$ not in a finite set of finite places.

51. A first consequence of strong local equidistribution is a "strong approximation" theorem: for a finite set of places $S$, let $U_{v}=\operatorname{supp}\left(\nu_{v}\right)$ for $v \in S$, and let $T_{v} \subset U_{v}$ be an open set. Then there exist infinitely many cusp forms $\pi \in \mathcal{A}(\mathbf{G})$ with $\pi_{v} \in T_{v}$ for all $v \in S$. This means that, although $\mathcal{A}(\mathbf{G})$ is an infinite set, one can specify fairly arbitrarily the behavior of the cusp forms in the family at any finite set of primes. ${ }^{(13)}$

Note that if we only have local equidistribution for certain places, we obtain a corresponding result for these places (individually).

52. If we consider the family of $L$-functions associated to a representation $\varrho$ of the $L$-group of $\mathbf{G}$ - assuming the minimal conditions in $\mathbf{2 5}$-, a soft "dominated convergence" argument leads to an asymptotic formula, with an Euler product as main term, far enough in the region of absolute convergence: for any fixed $s$ with $\operatorname{Re}(s) \geqslant \sigma_{0}$, where $\sigma_{0}$ may depend on $\varrho$ and the family, we have

$$
\mathbf{E}_{n}(L(\pi, \varrho, s)) \longrightarrow \prod_{v} \int_{\mathcal{A}_{v}} L_{v}(\theta, \varrho, s) d \nu_{v}(\theta)
$$

as $n \rightarrow+\infty$.

To see this, one simply notes that if we write

$$
L(\pi, \varrho, s)=\sum_{a} \lambda(a ; \pi, \varrho)(N a)^{-s}
$$

(where $a$ runs over non-zero integral ideals of $k$, with norm $N a$ ), each coefficient can be written as

$$
\lambda(a ; \pi, \varrho)(N a)^{-s}=\prod_{p \mid a} \lambda\left(p^{v_{p}(a)} ; \pi_{p}, \varrho\right)(N p)^{-v_{p}(a) s}
$$

which means it is a function in factorized form on $\mathcal{A}_{S}$, where $S$ is the set of primes $p$ dividing $a$. For fixed $a$, this is a bounded continuous function, and we can apply strong local equidistribution to obtain

$$
\mathbf{E}_{n}\left(\lambda(a ; \pi, \varrho)(N a)^{-s}\right) \longrightarrow(N a)^{-s} \prod_{p \mid a} \int_{\mathcal{A}_{p}} \lambda\left(p^{v_{p}(a)} ; \theta, \varrho\right) d \nu_{P}(\theta) .
$$

13. With quantitative forms of equidistribution, one can attempt to generalize this to certain unbounded sets of places; see [16, Th. 4] for an example. 
Applying dominated convergence, we can sum over a again to derive (10) using the multiplicativity in the form of the local formula

$$
\sum_{k \geqslant 0} \lambda\left(p^{k} ; \theta, \varrho\right)(N p)^{-k s}=L_{p}(\theta, \varrho, s) .
$$

Now, this is rather trivial from the point of view of analytic number theory, but one should perhaps take note that this applies simultaneously to all Langlands $L$-functions (when it makes sense) associated to the cusp forms in the family. This point illustrates an advantage of looking at equidistribution at the level of the cusp forms, and not of the $L$-functions. Another similar point is explained at the end of the text in $\mathbf{1 0 9 .}$

\section{Quantitative local equidistribution: level of decay}

53. In order to study more deeply the averages involving extended families of cusp forms, it is natural to expect that quantitative forms of local equidistribution are required. This may be formulated in different ways, but the goal is to have an analogue of the "level of distribution" in sieve theory.

54. Consider an extended family $\left(\mu_{n}\right)_{n}$ of cusp forms in $\mathcal{A}(\mathbf{G})$, satisfying strong local equidistribution (at the finite places, in the number field case), with limit measures $\nu_{v}$. For a continuous bounded function $\phi$ on $\mathcal{A}_{S}=\prod_{v \in S} \mathcal{A}_{v}$, denoting by $\nu_{S}$ the product over $v \in S$ of the $\nu_{v}$, we write

$$
\mathbf{E}_{n}(\phi(\pi))=\int_{\mathcal{A}_{S}} \phi(\theta) d \nu_{S}(\theta)+\varepsilon_{n}(\phi),
$$

which introduces the error term $\mathcal{E}_{n}(\phi)$.

55. Strong local equidistribution means of course that $\mathcal{E}_{n}(\phi) \rightarrow 0$ for any fixed $\phi$. In order to make this quantitative, we use the quantity $\kappa(n)$ defined in (1) to measure the complexity of cusp forms in the small families $\mu_{n}$, though it is conceivable that other parameters might be better adapted in certain situations.

If there is a system of norms $\|\cdot\|_{S}$ which is defined for functions on $\mathcal{A}_{S}$ for all $S$, one can say that $\left(\mu_{n}\right)$ has level of decay $\delta$ with respect to this choice of norms, for some $\delta>0$, if

$$
\left|\mathcal{E}_{n}(\phi)\right| \ll \kappa(n)^{-\delta}\|\phi\|_{S},
$$

with an absolute implied constant. It is enough to check such an inequality for factorized functions

$$
\phi\left(\left(\pi_{v}\right)_{v \in S}\right)=\prod_{v \in S} \phi_{v}\left(\pi_{v}\right)
$$

(which are quite often general enough for applications anyway.)

56. In practice, the inequality (12) might be proved for factorized functions $\phi$ which are of a very special form. Typically, $\phi_{v}$ is taken from an orthonormal basis of the Hilbert space $L^{2}\left(\mathcal{A}_{v}, \nu_{v}\right)$, containing the constant function 1 . This means that one proves strong local equidistribution, using the Weyl criterion, by showing

$$
\mathcal{E}_{n}(\phi) \ll \kappa(n)^{-\delta} \prod_{v \in S}\|\phi\|_{v}
$$


for factorized functions where each factor $\phi_{v}$ is in a fixed basis of the space of functions $\mathcal{A}_{v}$ with average value $0,{ }^{(14)}$ and the implied constant is independent of $S$. Here the main term vanishes (and these cases of the Weyl criterion suffice because if some $\phi_{v}$ is constant, one can replace $S$ by a smaller set of primes.)

57. From (13), we obtain (12) for continuous functions which lie in the closure of the linear combinations of basis functions with respect to the norms defined using multiplicativity.

58. This may be quite a small space (see Section 13 for an example; the same happens for classical cusp forms), but we can expect that it contains all functions on the unramified spectrum $\mathcal{A}_{v}^{n r}$ which are characters of finite-dimensional representations of the $L$-group of $\mathbf{G}$, when the unramified spectrum is identified - by the Satake isomorphism - with the semisimple conjugacy classes in the $L$-group.

59. Quantitative local equidistribution is easy to prove for $\mathrm{GL}_{1}$, and was proved for $\mathrm{GL}_{2}$ over $\mathbf{Q}$ by Royer [19] (and Conrey-Duke-Farmer independently).

60. The most general, and rather impressive, known case of quantitative equidistribution is due Shin and Templier [24]. It concerns two types of extended families, based on sets of cusp forms (with some multiplicity) on $\mathbf{G}$ having discrete series as local components at infinity, where either the weight is fixed and the level grows, or the level is fixed and the weight grows. The proofs are based on the earlier work of Shin [23], and require a quantitative treatement of his argument based on the trace formula.

61. The reader may again safely skip now to the next section; we describe here how the quantitative local equidistribution leads to "sieve" inequalities for the extended family (see Section 3 for the context).

We suppose that we have an extended family $\left(\mu_{n}\right)$ with strong local equidistribution at finite places, in the following quantitative form (which is a variant of the above): for every place $v$, we assume given an orthonormal basis of continuous functions $\left(\varphi_{v, n}\right)_{n \geqslant 0}$ of $L^{2}\left(\nu_{v}\right)$, with $\varphi_{v, 0}=1$, such that for any $n_{v} \geqslant 0, m_{v} \geqslant 0$, we have

$$
\mathbf{E}_{n}\left(\prod_{v \in S} \varphi_{v, n_{v}} \overline{\varphi_{v, m_{v}}}\right)=\prod_{v \in S} \delta\left(n_{v}, m_{v}\right)+O\left(\kappa(n)^{-\delta}\left(\prod_{v \in S} N v\right)^{A\left(n_{v}+m_{v}\right)}\right)
$$

for any finite set $S$ of finite places and some fixed $A>0, \delta>0$.

62. The first basic sieve statement is the following: for all places $v$, select a function on $\mathcal{A}_{v}$ of the form

$$
\Phi_{v}(\pi)=\sum_{n \leqslant d} \alpha_{v, n} \varphi_{v, n}(\pi)
$$

(a kind of "spectral polynomial" of fixed degree). Then the $\nu_{v}$ average of $\Phi_{v}$ is therefore $\alpha_{v, 0}$, and the $L^{2}$-norm is

$$
\sum_{n \leqslant d}\left|\alpha_{v, n}\right|^{2}=\left|\alpha_{v, 0}\right|^{2}+\sigma_{v}^{2}
$$

say.

14. So that here $\mathcal{E}_{n}(\phi)=\mathbf{E}_{n}(\phi(\pi))$. 
A Rényi-type large sieve inequality expresses a bound on the variance of $\Phi\left(\pi_{v}\right)$ when $v$ varies, averaged over the family:

$$
\mathbf{E}_{n}\left(\left|\sum_{N v \leqslant x} \Phi_{v}\left(\pi_{v}\right)-\sum_{N v \leqslant x} \alpha_{v, 0}\right|^{2}\right) \ll \Delta \sum_{N v \leqslant x} \sum_{1 \leqslant n \leqslant d}\left|\alpha_{v, n}\right|^{2}=\Delta \sum_{N v \leqslant x} \sigma_{v}^{2},
$$

where $\Delta$ is a constant that can be estimated by

$$
\Delta \leqslant 1+\kappa(n)^{-\delta} d x^{B}
$$

for some $B$ (depending on $A$ and $d$; one can take $B=1+2 A d$ for instance, using the standard argument in [13], though better bounds might be possible in special cases). This is non-trivial as long as $d x^{B} \ll \kappa(n)^{\delta}$, and it provides therefore some control over unbounded sets of places. The Montgomery-type inequality looks more like sieve: given the data as above, as well as some parameters $\delta_{v}>0$, we have

$$
\mathbf{P}_{n}\left(\Phi_{v}\left(\pi_{v}\right) \leqslant \alpha_{v, 0}-\delta_{v} \text { for all } v \text { with } N v \leqslant x\right) \ll \Delta H^{-1},
$$

where $\Delta$ can be bounded as above, and

$$
H=\sum_{N a \leqslant x}^{b} \prod_{v \mid a} \frac{\delta_{v}^{2}}{\sigma_{v}^{2}}
$$

(the sum ranging over squarefree integral ideals of norm at most $x$ ). The proof is an adaptation of the one ${ }^{(15)}$ in $[\mathbf{1 4}, \S 2]$ (which also contains some more information about this topic in the case of classical cusp forms.)

\section{Symmetry types for the behavior of $L$-functions on the critical line}

63. A basic question underlying the search for a good notion of family of cusp forms is the definition (and determination) of a "symmetry type" for a family of $L$-functions associated to the family. ${ }^{(16)}$ The existence of such a symmetry type is part of the Katz-Sarnak philosophy (see $[\mathbf{1 1}, \mathbf{1 2}]$ ), together with the assumption that it should belong to a very small list of possibilities, related to Haar-distributed families of random matrices in classical compact groups. We explain in this section a heuristic for determining the right symmetry type in certain extended families satisfying (strong) local equidistribution, and show how the "lowlying zeros" statistics can be computed under some assumption on the level of distribution or decay for the family, and on the limiting measures $\nu_{v}$. We will also recall the interpretation of symmetry types in terms of random matrices, and how this drives the conjectures for critical moments of $L$-functions.

For simplicity, we only consider the case of number fields $k$ in the computations, although the principles extend to function fields (there is an example of family over function fields in Section 12).

15. The standard proofs of Montgomery's inequality in the classical theory of the large sieve can not be adapted here.

16. We emphasize that, although the more fundamental notion is that of a family of cusp forms, the symmetry type, on the other hand, is an invariant of the $L$-functions under consideration. 
Shin and Templier [24] develop the type of computations sketched below for their families and obtain the same conclusion concerning the determination of symmetry types (the account below is independent of their work, though the latter existed in draft form earlier).

64. The reader should be aware that there are "exotic" behavior in extended families, even with apparently excellent equidistribution properties. In some cases, this suggests that more general models of random matrices may be needed to account for the weight involved in the measures $\mu_{n}$. An enlightening example of this is considered in Section 13, and another example due to A. Steiger is discussed in 82. However, these cases (which are, to the author's knowledge, the only ones known) can still be understood in a rather nice way as related to families with well-defined Katz-Sarnak-type symmetry type, where the random matrix model can be understood by modification of the Haar measures on the corresponding compact classical groups.

65. We consider an extended family $\left(\mu_{n}\right)$ of probability measures with strong local equidistribution (at the finite places) with respect to $\nu_{v}$ (which are also probability measures). Let $\nu_{v}^{n r}$ be the restriction of $\nu_{v}$ to the unramified spectrum of $\mathbf{G}_{v}$, seen as a measure on the compact space $K$ of semisimple conjugacy classes in a maximal compact subgroup of the $L$-group ${ }^{L} \mathbf{G}$. We will begin with the following assumptions about the limiting behavior of $\nu_{v}$ as the norm $N v$ of $v$ tends to infinity:

- Most of the mass of $\nu_{v}$ is supported on the unramified spectrum, and in fact

$$
\nu_{v}^{n r}\left(\mathcal{A}_{v}\right)=1+O\left((N v)^{-1 / 2-\delta_{0}}\right)
$$

for some $\delta_{0}>0$;

- We have

$$
\lim _{N v \rightarrow+\infty} \nu_{v}^{n r}=\nu
$$

where $\nu$ is the probability Haar measure on $K$ (see 78 for a discussion of more general situations, e.g, with $\nu_{v}^{n r}$ converging to another limit, or converging only on average over $v \ldots)$

For instance, the first condition holds if $\nu_{v}$ is supported on the unramified spectrum.

66. We come back to our earlier examples. For the measure $\nu_{p}$ on characters (for $k=\mathbf{Q}$, $\mathbf{G}=\mathrm{GL}_{1}$ ) described in $\mathbf{3 6}$, we see that the total measure of the ramified characters is $\ll p^{-1}$. The limit of $\nu_{p}$ is the uniform probability measure on the space $\mathbf{S}^{1}$ of unramified characters. For classical cusp forms, the Plancherel measures $\nu_{p}$ of (5) and their limit, the fixed Sato-Tate measure (6), also provide an example of (17) for families of classical modular forms.

More generally, for $\mathbf{G}=\mathrm{GL}_{n}$, the Plancherel measures $\nu_{v}^{P}$ on the unramified dual in $\mathcal{A}_{v}\left(\mathrm{GL}_{n}\right)$ satisfy (17) when suitably normalized, as in [1], where this can be checked. (Indeed, as explained to the author by A. Venkatesh, this holds for all reductive groups $\mathbf{G}$, at least split, and can be deduced from the formula of MacDonald for the density of the unramified $p$-adic Plancherel measure.)

67. Here comes a crucial point: essentially, any non-trivial level of decay allows the computation of low-lying zero distribution for the L-functions for restricted test functions. We explain this in the next few items.

68. We fix a non-trivial representation $\varrho$ of the $L$-group, as before. We will consider the distribution of zeros of the $L$-functions $L(\pi, \varrho, s)$, which are assumed to satisfy the standard 
analytic properties of $L$-functions (including functional equation). When $\mathbf{G}=\mathrm{GL}_{n}$, one can think of $\varrho$ as being the identity map, and therefore $L(\pi, \varrho, s)$ is just the standard $L$-function of degree $n$. As before, we denote by $\mathfrak{q}\left(\varrho_{*} \pi\right)$ the analytic conductor of $L(\pi, \varrho, s)$.

69. We first fix $\pi$, and assume (for simplicity; it is known that some unconditional statements can be made) the Riemann Hypothesis for $L(\pi, \varrho, s)$, and denote its zeros by

$$
1 / 2+i \gamma_{\pi, \varrho, j}, \quad j \in \mathbf{Z}
$$

(ordered with multiplicity and according to modulus, with $j \geqslant 0$ corresponding to zeros $\left.\gamma_{\pi, \varrho, j} \geqslant 0\right)$. Further, write

$$
-\frac{L^{\prime}}{L}(\pi, \varrho, s)=\sum_{a} \Lambda(a) c(a ; \pi, \varrho)(N a)^{-s}
$$

where $\Lambda$ is the von Mangoldt function of $k$. For $a=p^{k}$ with $p$ prime and $\pi$ unramified at $p$, we have

$$
c\left(p^{k} ; \pi, \varrho\right)=\operatorname{Tr} \varrho\left(\Theta_{p}(\pi)^{k}\right)
$$

in terms of the conjugacy class $\Theta_{p}(\pi)$ in the $L$-group which parameterizes $\pi_{p}$.

Then for a Schwartz function $\varphi$ with $\hat{\varphi}$ of compact support, the classical "explicit formula" leads to

$$
\begin{aligned}
& \sum_{j} \varphi\left(\frac{\gamma_{\pi, \varrho, j}}{2 \pi} \log \mathfrak{q}\left(\varrho_{*} \pi\right)\right)=\int_{\mathbf{R}} \varphi(x) d x- \\
& \frac{1}{\log \mathfrak{q}\left(\varrho_{*} \pi\right)} \sum_{p} \sum_{k \geqslant 1}\left(c\left(p^{k} ; \pi, \varrho\right)+\overline{c\left(p^{k} ; \pi, \varrho\right)}\right)(\log N p) \hat{\varphi}\left(\frac{k \log N p}{\log \mathfrak{q}\left(\varrho_{*} \pi\right)}\right)(N p)^{-k / 2} \\
& +R(\pi),
\end{aligned}
$$

where $p$ runs over prime ideals, and $R(\pi)$ is an "error term" that involves the archimedean factors.

70. Now we perform average with respect to $\mu_{n}$ on both sides, and attempt to obtain an asymptotic expansion of the left-hand side, as a functional of $\varphi$. Under very general circumstances, the average of $R(\pi)$ goes to zero as $n \rightarrow+\infty$ (often, $R(\pi) \ll\left(\log \mathfrak{q}\left(\varrho_{*} \pi\right)\right)^{-1}$ and $\left.\mathfrak{q}\left(\varrho_{*} \pi\right) \rightarrow+\infty\right)$. Similarly, it follows usually "for free" from easy bounds that the contribution of all $k \geqslant 3$ tends to zero, leaving the terms $k=1$ and $k=2$ to consider, say $S_{n}$ and $T_{n}$.

71. The term $k=1$ is given by

$$
\sum_{p} \mathbf{E}_{n}\left(\frac{1}{\log \mathfrak{q}\left(\varrho_{*} \pi\right)}\{c(p ; \pi, \varrho)+\overline{c(p ; \pi, \varrho)}\} \hat{\varphi}\left(\frac{\log N p}{\log \mathfrak{q}\left(\varrho_{*} \pi\right)}\right)\right)(\log N p)(N p)^{-1 / 2} .
$$

72. We now assume that the conductor varies "smoothly enough" in the family so that the factors $\log \mathfrak{q}\left(\varrho_{*} \pi\right)$ can be pulled out of the average and replaced with $\log \kappa(n)$. ${ }^{(17)}$ Thus

$$
S_{n} \approx \frac{1}{\log \kappa(n)} \sum_{p} \mathbf{E}_{n}(c(p ; \pi, \varrho)+\overline{c(p ; \pi, \varrho)}) \hat{\varphi}\left(\frac{\log N p}{\log \kappa(n)}\right)(\log N p)(N p)^{-1 / 2}
$$

17. This is of course clear if the conductor is constant for the small family $\mu_{n}$. It can be quite a bit more tricky for families of elliptic curves, for instance. 
Now we want to apply local equidistribution at each $p$. We assume that for $\phi(\pi)=c(p ; \pi, \varrho)$, and its conjugate, the norm $\|\phi\|_{p}$ involved in (12) are bounded by some power of $p$, say $p^{A}$. Then the quantitative decay gives

$$
\left.\mathbf{E}_{n}(c(p ; \pi, \varrho)+\overline{c(p ; \pi, \varrho)})=\int_{\mathcal{A}_{v}}\{c(p ; \theta, \varrho)+\overline{c(p ; \theta, \varrho)})\right\} d \nu_{p}(\theta)+O\left(p^{A} \kappa(n)^{-\delta}\right) .
$$

If $\hat{\varphi}$ has support in $[-\alpha, \alpha]$ for some $\alpha>0$ (depending on $A$ and on $\delta$ ), the sum over $p$ of the remainder term is limited to $N p \leqslant \kappa(n)^{\alpha}$, and is therefore bounded by

$$
\kappa(n)^{-\delta} \kappa(n)^{\alpha(A+1 / 2)},
$$

and goes to 0 as $n \rightarrow+\infty$ if $\alpha$ is small enough (depending on $A$ and $\delta$ ).

Moreover, using (16), we can restrict the integral to the unramified spectrum by the same type of arguments. Therefore we get

$$
\left.S_{n} \approx \frac{1}{\log \kappa(n)} \sum_{p} \hat{\varphi}\left(\frac{\log N p}{\log \kappa(n)}\right) \frac{(\log N p)}{(N p)^{1 / 2}} \int_{\mathcal{A}_{v}}\{\operatorname{Tr}(\varrho(\theta))+\overline{\operatorname{Tr}(\varrho(\theta)})\right\} d \nu_{p}(\theta) .
$$

73. Now we assume that $\varrho$ is an irreducible representation of the $L$-group. ${ }^{(18)}$ Under this assumption, by (17) and character theory, we have

$$
\left.\int_{\mathcal{A}_{p}}\{\operatorname{Tr}(\varrho(\theta))+\overline{\operatorname{Tr}(\varrho(\theta)})\right\} d \nu_{p}(\theta) \rightarrow 0
$$

as $N p \rightarrow+\infty$. This is not enough to continue, so we assume that, for some $\delta_{1}>0$, we have

$$
\left.\int_{\mathcal{A}_{p}}\{\operatorname{Tr}(\varrho(\theta))+\overline{\operatorname{Tr}(\varrho(\theta)})\right\} d \nu_{p}(\theta) \ll(N p)^{-1 / 2-\delta_{1}},
$$

in which case, we obtain

$$
S_{n} \rightarrow 0
$$

as $n \rightarrow+\infty$.

Quite often, this assumption holds for the reason that the integral is zero. For instance, this is the case when $\nu_{p}$ is the Plancherel measure (on the unramified spectrum); this may also happen when there is a symmetry to the measure which allows some change of variable to give 0 as a result. In Section 13, we present an example found in [17] where (21) does not hold, for rather deep arithmetic reasons.

74. There remains to handle $T_{n}$, the contribution of squares of primes. The same types of arguments involving local equidistribution lead (more easily, in fact, since the sum of $1 / N p$ is barely divergent) to

$$
\left.T_{n} \approx \frac{1}{\log \kappa(n)} \sum_{p} \hat{\varphi}\left(\frac{2 \log N p}{\log \kappa(n)}\right) \frac{(\log N p)}{N p} \int_{\mathcal{A}_{p}^{n r}}\left\{\operatorname{Tr}\left(\varrho\left(\theta^{2}\right)\right)+\overline{\operatorname{Tr}\left(\varrho\left(\theta^{2}\right)\right.}\right)\right\} d \nu_{p}(\theta)
$$

18. For instance, $\varrho$ is the identity on $\mathrm{GL}_{n}$. 
Now the assumption

$$
\begin{aligned}
\left.\int_{\mathcal{A}_{p}}\left\{\operatorname{Tr}\left(\varrho\left(\theta^{2}\right)\right)+\overline{\operatorname{Tr}\left(\varrho\left(\theta^{2}\right)\right.}\right)\right\} d \nu_{p}(\theta)= & \\
& \left.\int_{\mathcal{A}_{p}}\left\{\operatorname{Tr}\left(\varrho\left(\theta^{2}\right)\right)+\overline{\operatorname{Tr}\left(\varrho\left(\theta^{2}\right)\right.}\right)\right\} d \nu(\theta)+O\left((N p)^{-\delta_{2}}\right)
\end{aligned}
$$

for some $\delta_{2}>0$ is enough to ensure that

$$
T_{n} \approx \frac{2 \mathrm{FS}(\varrho)}{\log \kappa(n)} \sum_{p} \hat{\varphi}\left(\frac{2 \log N p}{\log \kappa(n)}\right)(N p)^{-1}
$$

where

$$
\mathrm{FS}(\varrho)=\int_{K} \operatorname{Tr}\left(\varrho\left(\theta^{2}\right)\right) d \nu(\theta)=\mathrm{FS}(\bar{\varrho})
$$

is the Frobenius-Schur indicator of the representation $\varrho$ (equal to that of $\bar{\varrho}$ ).

Hence, by the Prime Number Theorem, we get

$$
T_{n} \rightarrow \mathrm{FS}(\varrho) \frac{\varphi(0)}{2} .
$$

75. We summarize: under the assumptions (16), (17), (21) and (22) - and possibly some more technical ones having to do with variation of the conductor in the family, etc - we get, for suitably small support of $\hat{\varphi}$, the limit

$$
\mathbf{E}_{n}\left(\sum_{j} \varphi\left(\frac{\gamma_{\pi, \varrho, j}}{2 \pi} \log \mathfrak{q}\left(\varrho_{*} \pi\right)\right)\right) \rightarrow \int_{\mathbf{R}} \varphi(x) d x-\frac{\mathrm{FS}(\varrho)}{2} \varphi(0)
$$

as $n \rightarrow+\infty$. The limit can be written

$$
\int_{\mathbf{R}} \varphi(x) d \sigma_{\varrho}(x)
$$

where

$$
d \sigma_{\varrho}(x)=d x-\frac{\mathrm{FS}(\varrho)}{2} \delta_{0}
$$

76. The three basic, classical, symmetry types arise then from the trichotomy concerning the Frobenius-Schur indicator:

- If $\operatorname{FS}(\varrho)=0$, then $\varrho$ is of unitary type, the Frobenius-Schur indicator is zero, and we obtain the measure $d \sigma_{\varrho}(x)=d \sigma_{U}(x)=d x$; this is called the unitary type.

- If $\operatorname{FS}(\varrho)=1$, i.e., $\varrho$ is of orthogonal type (this means that $\varrho$ may be factored through an orthogonal group, or equivalently, there is a $\varrho$-invariant non-degenerate symmetric bilinear form on the space of $\varrho$ ), we get the measure $d \sigma_{S p}=d x-\frac{1}{2} \delta_{0}$, corresponding to the symplectic type. (See below for the shift!)

- If $\operatorname{FS}(\varrho)=-1$, i.e., $\varrho$ is of symplectic type (this means that there is a $\varrho$-invariant nondegenerate alternating bilinear form on the space of $\varrho$ ), we get the measure $d \sigma_{O}=d x+\frac{1}{2} \delta_{0}$, corresponding to the orthogonal type. 
77. The labeling of the three symmetry types is due to the following fact, first observed by Katz-Sarnak: let $\mathcal{T} \in\{\mathrm{U}, \mathrm{Sp}, \mathrm{O}\}$; for $N \geqslant 1$, let $\mathcal{T}_{N}$ be the corresponding maximal compact classical group, with probability Haar measure $m_{N}$. Then for $N \rightarrow+\infty$ and for $\varphi$ with $\hat{\varphi}$ having support in $]-1,1[$, we have

$$
\mathbf{E}_{N}\left(\sum_{j} \varphi\left(\frac{\vartheta_{j}}{2 \pi} N\right)\right) \longrightarrow \int_{\mathbf{R}} \varphi(x) d \sigma_{\mathcal{T}}(x),
$$

where $e^{i \vartheta_{j}}$ are the eigenvalues of a matrix in $\mathcal{T}_{N}$ (with arguments ordered from $-\pi$ to $\pi$ ). In other words: the symmetry type associated to the family of $L$-functions by means of the measure $d \sigma_{\varrho}$ indicates that the zeros close enough to the central point $1 / 2$ behave - after scaling to have mean-spacing 1 - "like" the eigenvalues of large random matrices of type $\mathcal{T}$, scaled in the same manner.

(The "shift" between orthogonal and symplectic types, which arises from the minus sign on the right-hand side of the explicit formula (19), is reminiscent of similar "change of signs" between alternating and orthogonal forms on suitable cohomology groups; e.g., the middle cohomology of a curve carries a symplectic pairing, but the $H^{2}$ of a surface - which can be seen as a family of curves - carries a symmetric pairing.)

78. We see that, formally, the symmetry type can be bound from the local equidistribution by simply checking if (17) holds, and then computing the Frobenius-Schur indicator. More general situations can be handled very similarly. For instance, in Sarnak's Type (3) ("restrictions on $H$-type"), we would not expect (17), but instead $\nu_{v}^{n r}$ might converge (as $N v \rightarrow+\infty$ ) to the probability Haar measure on a maximal compact subgroup $K_{H}$ of the subgroup $H \subset \mathrm{GL}_{m}(\mathbf{C})$. Then the same computations apply with (1) the condition of irreducibility of $\varrho$ replaced by the irreducibility of the restriction of $\varrho$ to $H$ (for the treatment of $S_{n}$ ); (2) the value of $\mathrm{FS}(\varrho)$ replaced by the Frobenius-Schur indicator $\operatorname{FS}\left(\varrho \mid K_{H}\right)$.

Other examples along these lines are given in Section 12 (in $\mathbf{9 7}$, the limit as $N v \rightarrow+\infty$ is Haar measure on a group with two elements, and in 91, it is Haar measure on the conjugacy classes of a group isomorphic to $\mathfrak{S}_{n}$.)

We observe that in all cases we know of strong local equidistribution, the measures $\nu_{v}$ converge to some limit as $N v \rightarrow+\infty$ (in the sense that most of the mass is found on a "common" set, and that the restriction there converge), and that this limit can be identified with the image on the set of conjugacy classes of the probability Haar measure of a compact Lie group $K$. (This should be the same as the one defined by Langlands that Sarnak mentions when discussing his Type (3) families.) This important property applies even to the "exotic" baheviors already mentioned (see Section 13 especially.)

79. However, the "formal" symmetry type might fail to be reflected in the limit of

$$
\mathbf{E}_{n}\left(\sum_{j} \varphi\left(\frac{\gamma_{\pi, \varrho, j}}{2 \pi} \log \mathfrak{q}\left(\varrho_{*} \pi\right)\right)\right),
$$

even in extremely favorable circumstances, because of the failure of the estimate (21) (the failure of (22) might also occur, of course, but since it is a much weaker condition, it seems much more unlikely - the author does not know of an example.) 
More precisely, if

$$
\left.\int_{\mathcal{A}_{p}}\{\operatorname{Tr}(\varrho(\theta))+\overline{\operatorname{Tr}(\varrho(\theta)})\right\} d \nu_{p}(\theta) \asymp(N p)^{-\delta_{3}}
$$

with $0 \leqslant \delta_{3}<1 / 2$, the sequence $\left(S_{n}\right)$ is certainly going to diverge. But in the critical case where $\delta_{3}=1 / 2$, say

$$
\left.\int_{\mathcal{A}_{p}}\{\operatorname{Tr}(\varrho(\theta))+\overline{\operatorname{Tr}(\varrho(\theta)})\right\} d \nu_{p}(\theta) \sim \lambda_{p}(N p)^{-1 / 2}
$$

we obtain

$$
S_{n} \approx \frac{1}{\log \kappa(n)} \sum_{p} \hat{\varphi}\left(\frac{\log N p}{\log \kappa(n)}\right) \lambda_{p}(N p)^{-1 / 2} .
$$

If $\lambda_{p}$ is constant, or has non-zero average over primes, this expression is of the same type as that giving $T_{n}$. In particular, it can then be expected to add a contribution to the "second" term $-\frac{1}{2} \mathrm{FS}(\varrho) \delta_{0}$ in the distribution of low-lying zeros. This will be the case in the example of Section 13. Low-lying zeros can lie! It seems that the "right" way to determine the symmetry type (if it exists) is to use the "formal" one, based on analysis of the limit of the local measure $\nu_{v}$, and the relevant Frobenius-Schur indicator.

80. In Section 12 below, we present some further examples of determination of symmetry types for some families related to families of extensions of $\mathbf{Q}$ with Galois groups $\mathfrak{S}_{n}$, and to function fields.

81. We didn't make any comment on the assumption that $\varrho$ be irreducible in this discussion. This is because if $\varrho$ splits as a direct sum

$$
\varrho=\varrho_{1} \oplus \varrho_{2}
$$

we have $L(\pi, \varrho, s)=L\left(\pi, \varrho_{1}, s\right) L\left(\pi, \varrho_{2}, s\right)$, so that the analysis can be performed separately on the irreducible summands, before combining them together if need be. And, similarly, if $\varrho$ is trivial or has a trivial component, we simply obtain fixed additional factors $\zeta_{k}(s)$ in the $L$-function.

82. A. Steiger has considered the following subfamily of primitive Dirichlet characters of prime levels: fix a non-empty open interval $I \subset \mathbf{S}^{1}$, and for $\ell$ prime, let

$$
D_{I, \ell}=\left\{\chi \in D_{\ell} \mid \varepsilon(\chi) \in I\right\}
$$

where $\varepsilon(\chi)$ is the "root number" of $L(s, \chi)$ (i.e., a normalized Gauss sum.) This is meant to be a "continuous" analogue of the restriction of a family to self-dual cusp forms with a given sign of functional equation. It is naturally suggested by the fact that $D_{\ell}$ satisfies strong local equidistribution, and that the root numbers of $\chi \in D_{\ell}$ are equidistributed on $\mathbf{S}^{1}$ with respect to Haar measure $\nu$ (as shown by Deligne using estimates for hyper-Kloosterman sums). Thus the cardinality of $D_{I, \ell}$ is asymptotically $(\ell-2) \nu(I)$ as $\ell$ tends to infinity.

Steiger shows that there is strong local equidistribution, with Haar measure on $\mathbf{S}^{1}$ as limiting measure for every prime $p$ (i.e., exactly like the situation for $D_{\ell}$ itself). Moreover, using Deligne's bound for hyper-Kloosterman sums, it is also possible to obtain a quantitative level of decay for the test functions $\phi(\theta)=\theta^{m}, m \in \mathbf{Z}$, on $\mathbf{S}^{1}$. The recipe above, for a family obeying Katz-Sarnak heuristic, would therefore suggest unitary symmetry type. This is confirmed for low-lying zeros using the quantitative equidistribution. 
Moreover, for the same reason, any statistic that can be computed from averages of values of the characters (e.g., 2-level density) will give the same answer as for the family $\left(D_{\ell}\right)$, and therefore as for random unitary matrices.

\section{Symmetry types and moments of $L$-functions}

83. The point of the determination of the symmetry type is, historically, that it is used in order to obtain the right conjectures for the behavior of moments of families of $L$-functions on the critical line. We recall the shape of these conjectures now, in the unitary and symplectic cases (see Section 11 for a discussion of the orthogonal case, where further distinctions are needed). Let $\left(\mu_{n}\right)$ be an extended family on $\mathrm{GL}_{n}, n \geqslant 1$, with formal symmetry type $\mathcal{T} \in\{\mathrm{U}, \mathrm{Sp}\}$. We consider the standard $L$-functions for simplicity. A minimal assumption is that the formal symmetry type should be reflected in the distribution of low-lying zeros (i.e., (23) holds for the right symmetry type), but this may not be sufficient (the family considered by Steiger, see 82, is a possible counter-example, which is currently being investigated).

84. The principles are: (1) one should model the $L$-values using matrices distributed according to Haar measure and taken from the symmetry type, together with a short Euler product at the small primes, which is distributed as dictated by local equidistribution ; (2) there is asymptotic independance of these two components - to a certain extent at least.

85. We continue with the setting of the previous section, in particular we consider $L$-functions $L(\pi, \varrho, s)$. The principles suggest that we compare

$$
\mathbf{E}_{n}\left(\left|L\left(\pi, \varrho, \frac{1}{2}\right)\right|^{2 m}\right)
$$

(for a fixed $m$ ) with

$$
\left(\int_{\mathcal{T}_{N}}|\operatorname{det}(1-g)|^{2 m} d m_{N}(g)\right)\left(\prod_{N p \leqslant x} \int_{\mathcal{A}_{p}}\left|L_{p}\left(\theta, \varrho, \frac{1}{2}\right)\right|^{2 m} d \nu_{p}(\theta)\right),
$$

for suitable $N$ and $x$. These parameters can be adjusted by doing the usual scaling of zeros and conductor, and by comparing the asymptotic behavior of the two factors (an easy matter for the Euler product, using arguments similar to those used in the explicit formula for lowlying zeros, and a fairly recent result of Keating-Snaith for the Random Matrix factor). Then, when $x=\log N$, the order of growth turns out to be the same, and the basic expectation is that, after renormalizing through this order of growth with $\log \kappa(n) \approx N$, one will obtain an asymptotic formula. Many partial results are known in this direction for certain families and suitable $m$.

86. We present the computations by distinguishing between the cases, motivated by the fact that the value at 1 of characteristic polynomials of symplectic or orthogonal matrices is a nonnegative real number. Consider first the symplectic type. We consider a fixed $m \in \mathbf{C}$ with $\operatorname{Re}(m) \geqslant 0$ (for instance, $m=i t$ with $t$ real, in which case we are looking at the "characteristic function", in the probabilistic language, of the random variable $\left.\log L\left(\pi, \varrho, \frac{1}{2}\right)\right)$. Then

$$
\int_{\mathrm{USp}(2 N)} \operatorname{det}(1-g)^{m} d m_{N}(g)=\left(\frac{N}{2}\right)^{\left(m^{2}+m\right) / 2} \Phi_{\mathrm{Sp}}(m)(1+o(1)),
$$


where

$$
\Phi_{\mathrm{Sp}}(m)=\frac{G(3 / 2)}{G(3 / 2+m)}
$$

as $N \rightarrow+\infty$ (this way of writing the limiting function $\Phi_{\mathrm{Sp}}(m)$ is the one described in [15], which is simpler than the one in Keating-Snaith).

Suppose $\varrho$ is $n$-dimensional, so $L(\pi, \varrho, s)$ is an $L$-function of degree $n$. The local $L$-function of a representation $\theta$ of $\mathrm{GL}_{n}\left(k_{p}\right)$ is always of the form

$$
L_{p}(\theta, s)=\prod_{1 \leqslant i \leqslant n} \frac{1}{1-\alpha_{i}(N p)^{-s}}
$$

where some $\alpha_{i}$ may possibly vanishing. We expand the $m$-th power of this in powers of $T=(N p)^{-s}$, getting

$$
L_{p}(\theta, s)^{m}=\sum_{k \geqslant 0} \lambda_{m}\left(p^{k} ; \theta\right)(N p)^{-k s}=1+\sum_{k \geqslant 1} \lambda_{m}\left(p^{k} ; \theta\right)(N p)^{-k s} .
$$

(for instance, if $m=1$, we have $\lambda_{1}\left(p^{k} ; \theta\right)=\lambda\left(p^{k} ; \theta\right.$, Id) with the notation in (11)).

Hence, if $\theta \in \mathcal{A}_{p}(\mathbf{G})$, we have

$$
L_{p}(\theta, \varrho, s)^{m}=1+\sum_{k \geqslant 1} \lambda_{m}\left(p^{k} ; \varrho_{*, p} \theta\right)(N p)^{-k s},
$$

For a fixed $m$, the functions $\theta \mapsto \lambda_{m}\left(p^{k} ; \varrho_{*, p} \theta\right)$ are bounded continuous functions on $\mathcal{A}_{p}(\mathbf{G})$, and hence

$$
\int_{\mathcal{A}_{p}} L_{p}\left(\varrho_{*} \theta, \frac{1}{2}\right)^{m} d \nu_{p}(\theta)=1+\sum_{k \geqslant 1}(N p)^{-k / 2} \int_{\mathcal{A}_{p}} \lambda_{m}\left(p^{k} ; \varrho_{*, p} \theta\right) d \nu_{p}(\theta) .
$$

Because of (16), the contribution of the ramified spectrum is $\ll(N p)^{-1-\delta_{0}}$ with $\delta_{0}>0$. For unramified $\theta \in \mathcal{A}_{p}$, look at the terms of low degree. We find by Taylor expansion that (in terms of the underlying representation $\varrho$ of the $L$-group) we have

$$
\lambda_{m}\left(p ; \varrho_{*, p} \theta\right)=m \operatorname{Tr} \varrho(\theta),
$$

and

$$
\lambda_{m}\left(p^{2} ; \varrho_{*, p} \theta\right)=\frac{m(m+1)}{2} \operatorname{Tr} \operatorname{Sym}^{2} \varrho(\theta)+\frac{m(m-1)}{2} \operatorname{Tr} \bigwedge^{2} \varrho(\theta) .
$$

Since the contribution of $k=1$ to the sum above is

$$
(N p)^{-1 / 2} \int_{\mathcal{A}_{p}} \lambda_{m}\left(p ; \varrho_{*, p} \theta\right) d \nu_{p}(\theta)=(N p)^{-1 / 2} \int_{\mathcal{A}_{p}} \operatorname{Tr} \varrho(\theta) d \nu_{p}(\theta),
$$

we see that under $(21)$, this is $\ll(N p)^{-1-\delta_{1}}$. The contribution of $k=2$ is

$$
\begin{aligned}
(N p)^{-1} \int_{\mathcal{A}_{p}} \lambda_{m}\left(p^{2} ; \varrho_{*, p} \theta\right) d \nu_{p}(\theta)= & (N p)^{-1}\left\{\frac{m(m+1)}{2} \int_{\mathcal{A}_{p}} \operatorname{Tr} \operatorname{Sym}^{2} \varrho(\theta) d \nu_{p}(\theta)\right. \\
& \left.+\frac{m(m-1)}{2} \int_{\mathcal{A}_{p}} \operatorname{Tr} \bigwedge \varrho(\theta) d \nu_{p}(\theta)\right\},
\end{aligned}
$$


and since (formal) symplectic symmetry means that $\varrho$ is irreducible with Frobenius-Schur indicator 1 , we see that the assumption that

$$
\begin{aligned}
\int_{\mathcal{A}_{p}} \operatorname{Tr}_{\operatorname{Sym}^{2}}^{2} \varrho(\theta) d \nu_{p}(\theta) & =\int_{\mathcal{A}_{p}} \operatorname{Tr} \operatorname{Sym}^{2} \varrho(\theta) d \nu(\theta)+O\left((N p)^{-\delta_{2}}\right), \\
\int_{\mathcal{A}_{p}} \operatorname{Tr} \bigwedge \varrho(\theta) d \nu_{p}(\theta) & =\int_{\mathcal{A}_{p}} \operatorname{Tr} \bigwedge \varrho(\theta) d \nu(\theta)+O\left((N p)^{-\delta_{2}}\right),
\end{aligned}
$$

(which is a refinement of (22), since the difference of the two integrals on the right is the Frobenius-Schur indicator) implies

$$
(N p)^{-1 / 2} \int_{\mathcal{A}_{p}} \lambda_{m}\left(p ; \varrho_{*, p} \theta\right) d \nu_{p}(\theta)=\frac{m(m+1)}{2} \frac{1}{N p}+O\left((N p)^{-1-\delta_{2}}\right) .
$$

By general principles, we therefore deduce that

$$
\prod_{N p \leqslant x} \int_{\mathcal{A}_{p}} L_{p}\left(\varrho_{*, p} \theta, \frac{1}{2}\right)^{m} d \nu_{p}(\theta)
$$

diverges like

$$
\prod_{N p \leqslant x}\left(1+\frac{m(m+1)}{2} \frac{1}{N p}\right) \asymp(\log x)^{m(m+1) 2}
$$

as $x$ grows, precisely

$$
\prod_{N p \leqslant x} \int_{\mathcal{A}_{p}} L_{p}\left(\varrho_{*, p} \theta, \frac{1}{2}\right)^{m} d \nu_{p}(\theta)=\prod_{N p \leqslant x}\left(1+\frac{m(m+1)}{2} \frac{1}{N p}\right) \Phi(m)(1+o(1))
$$

for each $m$ and for some function $\Phi$ (depending on the local equidistribution properties of the family; we refer to [15] and the references there for the discussion of the probabilistic structure of such a limit theorem.) This is similar to the Random Matrix estimate when $\log x=N / 2$. 87. In the unitary case, we only discuss the real part of $2 \log L\left(\pi, \varrho, \frac{1}{2}\right)$, which means the moments of the non-negative quantity $\left|L\left(\pi, \varrho, \frac{1}{2}\right)\right|^{2}$. Here, the Keating-Snaith asymptotic is

$$
\int_{U_{N}}|\operatorname{det}(1-g)|^{2 m} d m_{N}(g)=N^{m^{2}} \Phi_{\mathrm{U}}(m)(1+o(1)), \quad \Phi_{\mathrm{U}}(m)=\frac{G(1+m)^{2}}{G(1+2 m)},
$$

(valid for $\operatorname{Re}(m)>-1 / 2)$.

The arithmetic factor is investigated as before, writing

$$
\left|L_{p}(\pi, \varrho, s)\right|^{2 m}=\sum_{k \geqslant 0} \lambda_{m}\left(p^{k} ; r h o_{*} \pi\right)(N p)^{-k s}
$$

(with apologies for recycling notation) and using (16) to reduce to the unramified part of the spectrum. We find this time

$$
\begin{aligned}
& \lambda_{m}\left(p^{2} ; \varrho_{*, p} \theta\right)=m^{2} \operatorname{Tr}(\varrho \otimes \bar{\varrho})(\theta)+\frac{m(m+1)}{2}\left(\operatorname{Tr}_{\left.\operatorname{Sym}^{2} \varrho(\theta)+\operatorname{Tr} \operatorname{Sym}^{2} \bar{\varrho}(\theta)\right)+}\right. \\
& \frac{m(m-1)}{2}(\operatorname{Tr} \bigwedge \varrho(\theta)+\operatorname{Tr} \bigwedge \bar{\varrho}(\theta)) .
\end{aligned}
$$


Irreducibility of $\varrho$ means that

$$
\int_{\mathcal{A}_{p}}|\operatorname{Tr} \varrho(\theta)|^{2} d \nu(\theta)=1
$$

and we therefore need an additional bound

$$
\int_{\mathcal{A}_{p}}|\operatorname{Tr} \varrho(\theta)|^{2} d \nu_{p}(\theta)=1+O\left((N p)^{-\delta_{2}}\right)
$$

to derive (from the assumption of unitary symmetry, (21) and (26)) that

$$
1+\sum_{k \geqslant 1}(N p)^{-k / 2} \int_{\mathcal{A}_{p}} \lambda_{m}\left(p^{k} ; \varrho_{*, p} \theta\right) d \nu_{p}(\theta)=1+\frac{m^{2}}{N p}+O\left((N p)^{-1-\delta_{2}}\right),
$$

and therefore a rate of growth $(\log x)^{m^{2}}$ for the partial Euler product

$$
\prod_{N p \leqslant x} \int_{\mathcal{A}_{p}}\left|L_{p}\left(\theta, \varrho, \frac{1}{2}\right)\right|^{2 m} d \nu_{p}(\theta)
$$

in the case of unitary symmetry.

88. It would be interesting to have a proof of the asymptotic behavior of values of characteristic polynomials of random matrices which exhibits directly a link between the rate of growth (in (24) or (28)) and the Frobenius-Schur indicator. Those proofs that the author is aware of do not really explain this coincidence.

\section{Orthogonal symmetry types}

89. The orthogonal symmetry type is further split into three possibilities, corresponding to the classical groups $\mathrm{O}_{N}, \mathrm{SO}_{2 N}$ or $\mathrm{SO}_{2 N+1}$. The separation has to do with the property of the determinant \pm 1 , or rather with the value of $\operatorname{det}(-A)$, for $A \in \mathrm{O}_{N}$, which appears in the "functional equation"

$$
\operatorname{det}(1-T A)=\operatorname{det}(-A) \operatorname{det}(1-T A)
$$

of the reversed characteristic polynomial of an orthogonal matrix. Since $\operatorname{det}(-A)=$ $(-1)^{N} \operatorname{det}(A)$ for $A \in \mathrm{O}_{N}$, it becomes natural to separate even and odd matrix sizes to isolate random matrices with given "sign" of functional equation.

90. We record the asymptotic of moments for $\mathrm{SO}_{2 N}$ (orthogonal matrices of even size with "sign of functional equation" equal to 1$)$ : for $\operatorname{Re}(m)>1 / 2$, we have

$$
\int_{\mathrm{SO}(2 N)} \operatorname{det}(1-g)^{m} d m_{N}(g)=\left(\frac{N}{2}\right)^{\left(m^{2}-m\right) / 2} \Phi_{\mathrm{SO}}(m)(1+o(1)),
$$

where

$$
\Phi_{\mathrm{SO}}(m)=\frac{G(1 / 2)}{G(1 / 2+m)}
$$




\section{Other examples of symmetry types}

91. Another interesting example of determination of symmetry type is the following (which was also observed by N. Templier.) Fix an integer $n \geqslant 2$. We consider $L$-functions associated to an extension $K / \mathbf{Q}$ of degree $n$ with Galois closure having Galois group $\mathfrak{S}_{n}$ (which we just call "(S ${ }_{n}$-extensions" below). The Dedekind zeta function of $K$ is an Artin $L$-function for a permutation representation $\operatorname{Ind}_{G_{K}}^{G_{\mathbf{Q}}}(1)$ of dimension $n$. This splits as a direct sum

$$
\operatorname{Ind}_{G_{K}}^{G_{\mathbf{Q}}}(1)=1 \oplus \tilde{\varrho}_{K}
$$

of two irreducible representations, and we want to study the Artin $L$-functions $L\left(\tilde{\varrho}_{K}, s\right)$.

There should be some automorphic representation $\pi_{K} \in \mathcal{A}\left(\mathrm{GL}_{n-1}\right)$ with standard $L$-function given by $L\left(\tilde{\varrho}_{K}, s\right)$. Now consider the extended family of the $\pi_{K}$ 's associated to the finite sets $\left(\mathcal{F}_{X}\right)$ of all $\mathfrak{S}_{n}$-fields of discriminant $\leqslant X$.

The local component of $\pi_{K}$ at an unramified prime $p$ (for $K$ or for $\pi_{K}$, equivalently) is in the tempered unramified spectrum of $\mathrm{GL}_{n-1}\left(\mathbf{Q}_{p}\right)$. It corresponds therefore to a conjugacy class in $\mathrm{U}_{n-1}(\mathbf{C})$, and the compatibility of local $L$-factors means that this is the conjugacy class of $\varrho_{K}\left(\sigma_{p}\right)$, where $\sigma_{p}$ is the Frobenius at $p$.

One can show for $n \leqslant 4$, and one may expect more generally to be true, that there is local spectral equidistribution as $X \rightarrow+\infty$, with some limiting measure $\nu_{n, p}$ (necessarily of finite support, contained in the unramified spectrum, at least if $p>n$ ). A basic conjecture of Bhargava [4, Conj. 1.3] states that, as $p \rightarrow+\infty$, the measure $\nu_{n, p}$ converge to the image of the probability Haar measure of $\mathfrak{S}_{n}$ under the irreducible $(n-1)$-dimensional "augmentation" representation

$$
\tilde{\varrho}: \mathfrak{S}_{n} \longrightarrow \mathrm{U}_{n-1}(\mathbf{C}) \text {. }
$$

Bhargava's results (summarized in [4]; see also [3]) show that this is true for $n \leqslant 5$ (these are highly non-trivial facts!)

Now the formal symmetry type is easy to determine: we are basically looking at the restriction of the tautological representation of $\mathrm{GL}_{n-1}$ to the image of $\tilde{\varrho}$; the latter is irreducible, and has Frobenius-Schur indicator 1 (as all irreducible representations of $\mathfrak{S}_{n}$ do), hence the symmetry is of symplectic type.

92. If Bhargava's conjecture is correct, one can check that his prediction imply that

$$
\frac{1}{n !} \sum_{\sigma \in \mathfrak{S}_{n}} \operatorname{Tr}(\tilde{\varrho}(\sigma)) \nu_{n, p}(\sigma) \ll p^{-1}, \quad \frac{1}{n !} \sum_{\sigma \in \mathfrak{S}_{n}} \operatorname{Tr}\left(\tilde{\varrho}\left(\sigma^{2}\right)\right) \nu_{n, p}(\sigma)=1+O\left(p^{-1}\right),
$$

i.e., the analogues of $(21)$ and $(22)$ hold. So we expect the formal symmetry type to be reflected in the low-lying zeros of the family of $L\left(\pi_{K}, s\right)=\zeta_{K}(s) / \zeta(s)$. For $n=2$, this is easy to check, and for $n=3,4$, this was proved by Yang [25], building on quantitative forms of counting of cubic and quartic fields (due to Bellabas, Bhargava and Pomerance [3], and others).

93. We now come to another example. We included the case of all global fields in our basic definitions. This allows us to look at some concrete examples for functions fields over finite fields, where one can expect geometric insight and methods to lead to new results. 
94. Consider for instance $k=\mathbf{F}_{q}(T)$, the function field of the projection line over $\mathbf{F}_{q}$, and a family of elliptic curves over $k$, say in Weierstrass form

$$
E_{a, b}: y^{2}=x^{3}+a(t) x+b(t)
$$

where $a, b \in k$ vary in some algebraically-controlled way, say $(a, b) \in U\left(\mathbf{F}_{q}\right)$ for some algebraic variety $U / \mathbf{F}_{q}$ (e.g., $U_{d}$ which parameterizes polynomials of bounded bidegree $\leqslant d$ with cubic discriminant non-zero). For each $(a, b)$, we have a cusp form on $\mathrm{GL}_{2} / k$ associated to the elliptic curve (by results of Drinfeld), whose standard $L$-function is the $L$-function of $E_{a, b}$.

95. Extended families of two types arise naturally: either those associated with the finite sets $\left(U\left(\mathbf{F}_{q^{n}}\right)\right)_{n}$ for a fixed $U / \mathbf{F}_{q}$ (e.g., a fixed $\left.U_{d}\right)$, or those associated with $\left(U_{d}\left(\mathbf{F}_{q}\right)\right)_{d}$, where the base field $\mathbf{F}_{q}$ is fixed.

96. The former belong to the realm of algebraic geometry, and Deligne's Equidistribution Theorem, combined with the deep monodromy computations of Katz, leads to a very good understanding (indeed, this is where the Katz-Sarnak philosophy comes in.) Ironically, such families can not enter into the framework of this text, because the implied change of base field from $\mathbf{F}_{q}(T)$ to $\mathbf{F}_{q^{n}}(T)$ induces also changes in the set of places as $n$ varies, so that one can not "track" the distribution of the local representations at a fixed place.

97. The second type of extended families, however, are of similar difficulty as the number field case, though of course the possibility to rely on the Riemann Hypothesis is very useful! The following example of families of hyperelliptic curves is an example which has been the topic of a number of investigations (e.g., by Kurlberg-Rudnick [18], or Faifman-Rudnick [9]). One considers the varieties $U_{d} / \mathbf{F}_{q}$ (with $q$ fixed, odd) and $d$ odd (say) is the dense open subset of monic polynomials of degree $d$ in $\mathbf{F}_{q}[T]$ which are squarefree (i.e., have no repeated root in an algebraic closure of $\mathbf{F}_{q}$ ). Then we consider the family of smooth projective curve with affine model given by

$$
H_{f}: y^{2}=f(x)
$$

for $f \in U_{d}\left(\mathbf{F}_{q}\right)$. This set of curves "is" a small family of algebraic curves. The interesting part of the zeta function of $H_{f}$ is the $L$-function of the quadratic "Dirichlet" character $\chi_{f}$ of $\mathbf{F}_{q}[T]$ associated to $f$, and thus by varying $d$ we obtain an extended family of (quadratic) characters of $k=\mathbf{F}_{q}(T)$ (so that the group involved is $\mathrm{GL}_{1} / k$.)

For a place of $k$ given by a monic irreducible polynomial ${ }^{(19)} \pi \in \mathbf{F}_{q}[T]$, the local character at $\pi$ of $\chi_{f}$ is either the unramified quadratic character mapping $\pi$ (seen as uniformizer) to $\chi_{f}(\pi) \in\{ \pm 1\}$ (say $\varepsilon_{+}=1$ and $\varepsilon_{-}$), or - if $\pi$ divides $f$ - one of the two ramified quadratic characters $\eta_{ \pm}$. The asymptotic probabilities, as $d \rightarrow+\infty$, of these are easily computed and one finds that there is local equidistribution with limiting measure $\nu_{\pi}$ supported on the set of quadratic characters of $k$ with

$$
\nu_{\pi}\left(\varepsilon_{ \pm}\right)=\frac{1}{2} \frac{|\pi|}{1+|\pi|}, \quad \nu_{\pi}\left(\eta_{ \pm}\right)=\frac{1}{2} \frac{1}{1+|\pi|}
$$

where $|\pi|=q^{\operatorname{deg}(\pi)}$. Note that, as $|\pi|$ tends to infinity, the measure $\nu_{\pi}$ converges to the uniform measure on $\left\{\varepsilon_{ \pm}\right\} \simeq\{ \pm 1\}$.

19. We hope there will no difficulty in understanding that $\pi$ is used to denote polynomials in this section. 
In particular, we find that the first moment of $\nu_{\pi}$, as a measure on $\{ \pm 1\}$, is always 0 , and the second moment is $1-1 /(1+|\pi|) \rightarrow 1$ as $|\pi| \rightarrow+\infty$. These correspond, using the heuristics of Section 9, to a symplectic symmetry, which fits well with the fact that the moduli spaces of hyperelliptic curves of genus $g$ (and even suitable one-parameter subfamilies on it) have geometric monodromy group equal to the symplectic group $\mathrm{Sp}_{2 g}$ (see, e.g., [11, $\S 11$ ] for these computations.)

\section{An example with Siegel modular forms}

98. This section presents a particular example of families for the group $\mathrm{PGSp}_{4}$, corresponding to holomorphic Siegel modular forms. ${ }^{(20)}$ The results are due to Saha, Tsimerman and the author [17], and exhibit an interesting combination of unusual features:

- It is an example of a group of (semisimple) rank 2, like $\mathrm{GL}_{3}$; as we will explain, this has certain interesting consequences in the study of invariants of associated $L$-functions, which are not visible for groups of rank 1 (and are also hidden in the case of $\mathrm{GL}_{3}$ );

- But the group is not among the general linear groups $\mathrm{GL}_{n}$, and is probably the simplest example not of this type (note that, for $\mathrm{GSp}_{2 g}$, the $L$-group is usually not $\mathrm{GSp}_{2 g}(\mathbf{C}$ ), but a spin group; however, the connected component of identity of the $L$-group of PGSp 4 is isomorphic to $\operatorname{Spin}_{5}(\mathbf{C}) \simeq \operatorname{Sp}_{4}(\mathbf{C})$ (one of the exceptional isomorphisms of algebraic groups);

- The families used are not based on counting measures, but rather involve a special weight which makes it possible to apply analogues of the Petersson formula for classical holomorphic cusp forms;

- This weight turns out to involve global invariants of the corresponding cusp forms, which has the effect of bringing the asymptotic behavior of certain $L$-functions out of the range of the Katz-Sarnak symmetry types - at least, conjecturally;

- However, because the global invariant mentioned in the previous item is not immediately visible, the asymptotic behavior we obtain can be interpreted in turn as giving strong evidence for a beautiful conjecture of Böcherer concerning the arithmetic nature of Fourier coefficients of Siegel modular forms on $\mathrm{Sp}_{4}$.

99. For $k \geqslant 0$ even integer, let $\mathcal{S}_{k}$ be an orthogonal basis (for the Petersson inner product) of primitive forms of the space of Siegel cusp forms of weight $k$ with respect to $\operatorname{Sp}_{4}(\mathbf{Z})$, and let $\mathcal{S}_{k}^{*} \subset \mathcal{A}\left(\mathrm{PGSp}_{4}\right)$ be the corresponding set of cuspidal representations of $\mathrm{PGSp}_{4}$ (note that the basis $\mathcal{S}_{k}$ is not known to be unique). For $f \in \mathcal{S}_{k}$ and a matrix $t \in M_{2}(\mathbf{Q})$ which is half-integral, symmetric and positive definite, we denote by $a_{f}(t)$ the corresponding Fourier coefficient. Then we define

$$
\omega_{k}(f)=c_{k} \frac{\left|a_{f}(1)\right|^{2}}{\langle f, f\rangle}, \quad c_{k}=\frac{\pi}{4}(4 \pi)^{3-2 k} \Gamma(k-3 / 2) \Gamma(k-2)
$$

20. Just as classical modular forms involving $\mathrm{SL}_{2}(\mathbf{Z})$ correspond, representation-theoretically, to PGL 2 , so the Siegel cusp forms are related to the group $\mathrm{PGSp}_{4}$, the quotient of $\mathrm{GSp}_{4}$ by its center. One could also work at the level of $\mathrm{GSp}_{4}$ itself. 
(where 1 corresponds to the identity matrix), and we finally define extended families $\left(\mu_{k}\right)$ such that $\mathcal{S}_{k}^{*}$ is the support of $\mu_{k}$ and

$$
\mu_{k}(\{\pi\})=\sum_{\pi(f)=\pi} \omega_{k}(f)
$$

where $\pi \in \mathcal{S}_{k}^{*}$ and $\pi(f)$ is the automorphic representation "generated" by $f$ (multiplicity one is not known, so that different f's might lead to the same representation, and we take this possible multiplicity into account). We use the tautological representation ${ }^{L} \mathrm{PGSp}_{\mathbf{4}}=$ $\mathrm{Sp}_{4}(\mathbf{C}) \hookrightarrow \mathrm{GL}_{4}(\mathbf{C})$ here to define the analytic conductor of $\pi \in \mathcal{S}_{k}^{*}$, which gives $\tilde{\mathfrak{q}}(\pi)=k^{2}$, and hence $\kappa(n)=k^{2}$ in this case.

This definition looks superficially similar to that of families of classical cusp forms involving the Petersson norm as a weight. The main difference - crucial, as it turns out - is that the factor $\left|a_{f}(1)\right|^{2}$ can be normalized to be 1 by the Atkin-Lehner theory of newforms on $\mathrm{GL}_{2}$, which is not quite possible here.

100. The local unramified spectrum of $\mathrm{PGSp}_{4}\left(\mathbf{Q}_{p}\right)$ ( since the level is 1, we need not consider the ramified spectrum) can be parameterized by two non-zero complex numbers $(a, b)$, modulo the action of the Weyl group $W$, which amounts to saying that a function on $\mathcal{A}_{p}$ is a function $\phi(a, b)$ which is symmetric, and invariant under replacing $a$ by $a^{-1}$, or $b$ by $b^{-1}$ (or both). The tempered unramified spectrum is then identified with $(a, b) \in \mathbf{S}^{1} \times \mathbf{S}^{1}$, modulo the same identification. It can be identified with the space of conjugacy classes in the compact unitary symplectic group $K=\mathrm{USp}_{4}(\mathbf{C})$, by sending a matrix with eigenvalues $\left(a, b, a^{-1}, b^{-1}\right)$ to $a, b$.

101. The main results of [17] concerning local equidistribution ${ }^{(21)}$ are:

1. The extended family $\left(\mu_{k}\right)_{k}$ satisfies strong local equidistribution at finite places; the limiting measure $\nu_{p}$, are supported on the tempered unramified spectrum (so (16) holds). They depend on $p$, and are the so-called Bessel-Plancherel measures of Furusawa-Shalika (associated to the choice of the Bessel model corresponding to the Fourier coefficient at 1).

2. These have explicit - but somewhat unwieldy, depending on the splitting of $p$ in $\mathbf{Q}(i)$ - descriptions in terms of the coordinates $(a, b)$ above. At least, these show straightforwardly that, as $p$ tends to infinity, the measures $\nu_{p}$ converge to the probability Haar measure $\nu$ on $K=\mathrm{USp}_{4}(\mathbf{C})^{\sharp}$, i.e., the property (17) also holds for this extended family.

3. The equidistribution holds quantitatively in the following sense. First, for any prime $p$, there is a natural orthonormal basis of $L^{2}\left(\mathcal{A}_{p}, \nu_{p}\right)$ of (restrictions and projections of) Laurent polynomials in two variables, denoted $\left(U_{p}^{l, m}\right)_{l, m \geqslant 0}$, which are invariant under $W$. For any finite set of primes $S$, and any $S$-tuples $\boldsymbol{l}$ and $\boldsymbol{m}$, we have

$$
\mathbf{E}_{k}\left(\prod_{p \in S} U_{p}^{l_{p}, m_{p}}\left(\pi_{p}\right)\right)=\Delta(\boldsymbol{l} ; \boldsymbol{m})+O\left(k^{-2 / 3} \prod_{p \in S} p^{l_{p}+3 m_{p} / 2+\varepsilon}\right)
$$

for any $\varepsilon>0$, where the main term $\Delta(\boldsymbol{l} ; \boldsymbol{m})$ is zero unless both $\boldsymbol{l}$ and $\boldsymbol{m}$ are 0 . Note that $k^{-2 / 3}=\kappa(n)^{-1 / 3}$, so this is of the form (12).

21. There are other applications and more general versions in the paper. 
102. We apply these facts to families of $L$-functions. The $L$-group $\operatorname{Sp}_{4}(\mathbf{C})$ has two fundamental representations, the 4-dimensional embedding

$$
\varrho_{1}: \mathrm{Sp}_{4}(\mathbf{C}) \hookrightarrow \mathrm{GL}_{4}(\mathbf{C}),
$$

and the 5-dimensional "projection" that comes from the composition

$$
\varrho_{2}: \operatorname{Sp}_{4}(\mathbf{C}) \simeq \operatorname{Spin}_{5}(\mathbf{C}) \longrightarrow \mathrm{SO}_{5}(\mathbf{C}) \hookrightarrow \mathrm{GL}_{5}(\mathbf{C}) .
$$

We denote by or $L\left(\pi, \varrho_{1}, s\right)$ or simply $L(\pi, s)$ (respectively $L\left(\pi, \varrho_{2}, s\right)$ ) the corresponding $L$ function for a cusp form $\pi \in \mathcal{A}\left(\mathrm{PGSp}_{4}\right)$. For the first $L$-function (which is also called the spinor $L$-function), Andrianov and others have proved all standard analytic properties. For the second, these properties have been proved by Böcherer and Mizumoto. Very recently, Pitale, Saha and Schmidt have proved that there exists a well-defined Langlands functoriality map

$$
\varrho_{1, *}: \mathcal{A}\left(\mathrm{GSp}_{4}\right) \longrightarrow \mathcal{A}\left(\mathrm{GL}_{4}\right)
$$

so that one can see the spinor $L$-function as a standard $L$-function on $\mathrm{GL}_{4}$.

In terms of the parameters $(a, b)$, the local $L$-functions at $p$ for these $L$-functions are given by

$$
\left(1-a p^{-s}\right)^{-1}\left(1-b p^{-s}\right)^{-1}\left(1-a^{-1} p^{-s}\right)^{-1}\left(1-b^{-1} p^{-s}\right)^{-1},
$$

and

$$
\left(1-p^{-s}\right)^{-1}\left(1-a b p^{-s}\right)^{-1}\left(1-a^{-1} b p^{-s}\right)^{-1}\left(1-a b^{-1} p^{-s}\right)^{-1}\left(1-a^{-1} b^{-1} p^{-s}\right)^{-1}
$$

respectively. ${ }^{(22)}$

103. For the family of spinor $L$-functions, applying the recipe in $\mathbf{7 8}$, we know that the representation $\varrho_{1}$ is irreducible and is of symplectic type, suggesting a (formal) orthogonal symmetry type. However, when it comes to actually determining the distribution of low-lying zeros, we find

$$
\int_{K} \operatorname{Tr}\left(\varrho_{1}(\theta)\right) d \nu_{p}(\theta)=\frac{\lambda_{p}}{\sqrt{p}},
$$

where $\lambda_{p}$ is the number of ideals of norm $p$ in $\mathbf{Z}[i]$, and

$$
\int_{K} \operatorname{Tr}\left(\varrho_{2}\left(\theta^{2}\right)\right) d \nu_{p}(\theta)=-1+O\left(p^{-1}\right),
$$

which means that (22) holds, but not (21). In fact, this is the "critical" case mentioned in 79. The average value of $\lambda_{p}$ is one, and one computes that this leads (together with the quantitative equidistribution result) to a distribution of low-lying zeros $1 / 2+i \gamma_{f, j}$ given by

$$
\mathbf{E}_{k}\left(\sum_{j} \varphi\left(\frac{\gamma_{f, j}}{2 \pi} \log \mathfrak{q}(f)\right)\right) \longrightarrow \int_{\mathbf{R}} \varphi(x) d x-\frac{1}{2} \varphi(0)
$$

for $\varphi$ with $\hat{\varphi}$ having support in ] $-4 / 15,4 / 15[$. This is the same as would be obtained for a symplectic symmetry type!

22. More generally, for Siegel cusp forms on $\mathrm{GSp}_{2 g}, g \geqslant 2$, one has similarly a spinor $L$-function of dimension $2^{g}$, and a "projection" one of dimension $2 g+1$, corresponding to the spin representation of $\operatorname{Spin}_{2 g+1}(\mathbf{C})$ and its projection to the $L$-group $\mathrm{SO}_{2 g+1}(\mathbf{C})$; see [2] for a discussion of their properties. 
104. It should be noted that the work of Shin [23] and the developments of Shin and Templier [24] should provide a proof that, omitting the weight $\omega_{k}(f)$, one obtains an extended family where the formal symmetry type is reflected in the low-lying zeros.

105. The fact that the low-lying zeros in our extended family fail to reflect the formal symmetry type is interpreted in [17] using a beautiful conjecture of Böcherer, as refined by Furusawa, Shalika, Martin and others. This conjecture suggests that the weight $\omega_{k}(f)$ used in the definition of the measure $\mu_{k}$ carries arithmetic information, through the Fourier coefficient $\left|a_{f}(1)\right|^{2}$, and in fact carries critical values of $L$-functions. Precisely, one expects that

$$
\omega_{k}(f) \simeq \frac{L\left(f, \frac{1}{2}\right) L\left(f \times \chi_{-4}, \frac{1}{2}\right)}{\langle f, f\rangle}
$$

where $\chi_{-4}$ is the real primitive character of conductor 4 (the notation $\simeq$ indicates that there would be extra factors, depending on $k$ and maybe on $f$, but "independent" of the spinor $L$ values). One can expect that $L\left(f \times \chi_{-4}, \frac{1}{2}\right)$ behaves independently of $L(f, s)$, after averaging. However, this is obviously not so for the factor $L\left(f, \frac{1}{2}\right)$ itself. ${ }^{23)}$

106. In [17, End of $\S 5.4]$, it is shown how assuming this conjecture, as well as some averaging properties on $\mathcal{S}_{k}$ without the weight $\omega_{k}(f)$ (i.e., roughly, assuming that the orthogonal "formal" symmetry type is correct), one can recover the formula (32). Since the latter is a theorem, we interpret this as some global evidence for the strong form of Böcherer's conjecture described above. Indeed, at the very least, it shows that $\left|a_{f}(1)\right|^{2} /\langle f, f\rangle$ correlates quite strongly with the spinor $L$-function.

107. A fairly natural random matrix model of the spinor $L$-values (up to fitting of parameters) is given here by the "size-biased" characteristic polynomial of a matrix in $\mathrm{SO}_{2 N}$, i.e., by looking at the measures $(24)$

$$
d \tilde{m}_{N}(g)=\frac{1}{2} \operatorname{det}(1-g) d m_{N}(g)
$$

on $\mathrm{SO}_{2 N}$. The factor $1 / 2$ is such that these are probability measures. Because matrices with $\operatorname{det}(1-g)$ small are deemphasized, the distribution of $\operatorname{det}(1-g)$ is, with respect to this measure, altered so as to show greater probability of large values. To be precise, one can show (see [7, §3.7, Th. 12]) that the moments behave almost like those of symplectic matrices, i.e., we have

$$
\int_{\mathrm{SO}_{2 N}} \operatorname{det}(1-g)^{m} d \tilde{m}_{N}(g)=\left(\frac{N}{2}\right)^{m(m+1) / 2} \Psi(m)(1+o(1))
$$

for some limiting function $\Psi$ (which closely resembles $\Phi_{\mathrm{Sp}}(m)$, but is not exactly the same). Similarly, analyzing the Euler product of local Spinor $L$-functions

$$
\prod_{p \leqslant x} \int_{\mathcal{A}_{p}} L_{p}\left(\theta ; \frac{1}{2}\right)^{m} d \nu_{p}(\theta)
$$

\footnotetext{
23. This factor is not present in the original conjecture of Böcherer, which concentrates on the variation of Fourier coefficients $a_{f}(t)$ with the discriminant of $t$; this corresponds here to the factor $L\left(f \times \chi_{-4}, \frac{1}{2}\right)$.

24. The Böcherer conjecture as stated above suggests adding another factor to account for the twisted $L$ valued, but there are good reasons to think of it as (probabilistically) independent, and therefore contributing only a normalizing factor.
} 
(as in 86), we find that it diverges like

$$
(\log x)^{m(m+1) / 2}=\log (x)^{m(m-1) / 2+m},
$$

one factor $m$ coming from the contribution of $k=1$, using (30).

108. It would be interesting to have more evidence for (or against) the use of such a matrix model for this family of Siegel cusp forms and their spinor $L$-values. Indeed, agreement would be a strong argument in favor of the existence of actual "matrices" becoming equidistributed "somewhere" (from which the average of $L$-values on the critical line arise), instead of there being only some kind of "normal form" (or universality) explanation for the coincidence of formulas between Random Matrix Theory and $L$-functions. (Note that there is more evidence for this, e.g., the recent work of Dueñnez, Huynh, Keating, Miller and Snaith [8] on modeling $L$-values of elliptic curves for bounded, non-asymptotic, conductors.)

109. Here is a last remark about the distinction between families of cusp forms and of $L$ functions: the Dirichlet expansion of the $L$-function (used also in the "arithmetic" factor for moment conjectures on the critical line) and the determination of the symmetry type involve functions of the local parameters, that we called $\lambda(a ; \pi, \varrho)$ or $\lambda_{m}(a ; \pi)$ in $(11),(25)$ and $c\left(p^{k} ; \pi, \varrho\right)$ in (18). Each of them are characters of virtual representations of the $L$-group ${ }^{L} \mathbf{G}$ (possibly with $\mathbf{C}$-coefficients), the $c$-coefficients reflecting the Adams or $\Psi$-operations on the representation ring.

The functions $\pi \mapsto \lambda(a ; \pi, \varrho)$ are enough to express the Dirichlet series representing $L(\pi, \varrho, s)$, and one may think that understanding the equidistribution properties of these functions on the spectrum would suffice to understand much of the behavior of $L(\pi, \varrho, s)$, at least conjecturally. However, in the example of Siegel cusp forms, one sees that the coefficient $c\left(p^{2} ; \pi, \varrho_{1}\right)$ occuring in the determination of the low-lying zeros of the spinor $L$-functions is closely related to the coefficient $\lambda\left(p ; \pi, \varrho_{2}\right)$ of the projection $L$-function. This coefficient is linearly independent from all the functions $\lambda\left(p^{k} ; \pi, \varrho_{1}\right.$ ) (see $[\mathbf{1 7},(2.3 .7)]$, in the limit $p \rightarrow+\infty$ which gives the Haar measure on $\mathrm{USp}_{4}(\mathbf{C})$, as already discussed). Hence even to understand a single type of L-functions, it may be essential to understand equidistribution for other L-functions of the underlying cusp forms!

\section{References}

[1] A-M. Aubert and R. Plymen: Plancherel measure for $G L(n, F)$ and $G L(m, D)$ : explicit formulas and Bernstein decomposition, J. Number Theory 112 (2005), 26-66.

[2] M. Asgari and R. Schmidt: Siegel modular forms and representations, Manuscripta Math. 104 (2001), 173-200.

[3] K. Bellabas, M. Bhargava and C. Pomerance: Error estimates for the Davenport-Heilbronn theorems, Duke Math. J. 153 (2010), pp. 173-210.

[4] M. Bhargava: Mass formulae for extensions of local fields, and conjectures on the density of number field discriminants, Internat. Math. Res. Notices (2007).

[5] J. Cogdell and P. Michel: On the complex moments of symmetric power L-functions at $s=1$, Internat. Math. Res. Notices (2004), 1561-1617.

[6] J.B. Conrey, D. Farmer, J. Keating, M. Rubinstein and N. Snaith: Integral moments of Lfunctions, Proc. Lond. Math. Soc. 91 (2005) 33-104. 
[7] F. Delbaen, E. Kowalski and A. Nikeghbali: Mod- $\varphi$ convergence, preprint (2011), arXiv:1107. 5657.

[8] E. Dueñez, D.K. Huynh, J.P. Keating, S.J. Miller, and N.C. Snaith: A random matrix model for elliptic curve L-functions of finite conductor, J. Phys. A: Math. Theor. 45 (2012), 115-207; arXiv: 1107.4426.

[9] D. Faifman and Z. Rudnick: Statistics of the zeros of zeta functions in families of hyperelliptic curves over a finite field, Compositio Math. 146 (2010), 81-101; arXiv:0803.3534.

[10] H. Iwaniec and E. Kowalski: Analytic number theory, A.M.S Coll. Publ. 53, A.M.S (2004).

[11] N. Katz and P. Sarnak: Random matrices, Frobenius eigenvalues, and monodromy, A.M.S Coll. Publ., A.M.S (1999).

[12] N. Katz and P. Sarnak: Zeroes of zeta functions and symmetry, Bull. Amer. Math. Soc. 36 (1999), $1-26$.

[13] E. Kowalski: The large sieve and its applications: arithmetic geometry, random walks, discrete groups, Cambridge Tracts in Math. 175, Cambridge Univ. Press (2008).

[14] E. Kowalski: Amplification arguments for large sieve inequalities, Archiv der Math. 94 (2010), 443-457.

[15] E. Kowalski and A. Nikeghbali: Mod-Gaussian convergence and the value distribution of $\zeta(1 / 2+$ it) and related quantities, Journal of the London Math. Soc. 86 (2012), 291-319.

[16] E. Kowalski, K. Soundararajan, Y.K. Lau and J. Wu: On modular signs, Math. Proc. Cambridge Phil. Soc. 149 (2010), 389-411.

[17] E. Kowalski, A. Saha and J. Tsimerman: Local spectral equidistribution for Siegel modular forms and applications, Compositio Math. 148 (2012), 335-384.

[18] P. Kurlberg and Z. Rudnick: The fluctuations in the number of points on a hyperelliptic curve over a finite field, Journal Number Th. 129 (2009), 580-587; arXiv:0804.0808.

[19] E. Royer: Facteurs Q-simples de $J_{0}(N)$ de grande dimension et de grand rang, Bull. Soc. Math. France 128 (2000), 219-248.

[20] P. Sarnak: Definition of families of L-functions, letter to H. Iwaniec, P. Michel, A. Venkatesh and others (2008); http://publications.ias.edu/sarnak/paper/507

[21] P. Sarnak: Statistical properties of eigenvalues of the Hecke operator, in Analytic Number Theory and Diophantine Problems, Progress in Math. 60, Birkhäuser, 1987; 75-102.

[22] J-P. Serre: Répartition asymptotique des valeurs propres de l'opérateur de Hecke $T_{p}$, J. American Math. Soc. 10 (1997), 75-102.

[23] S-W. Shin: Plancherel density theorem for automorphic representations, Israel J. Math. v.192 (2012) 83-120.

[24] S-W. Shin and N. Templier: Sato-Tate theorem for families and low-lying zeros of automorphic L-functions, preprint (2012); arXiv:1208.1945.

[25] A. Yang: Low-lying zeros of Dedekind zeta functions attached to $S_{4}$ quartic fields, preprint (2010).

12 février 2013

E. KowAlski, ETH Zürich - DMATH, Rämistrasse 101, 8092 Zürich, Switzerland E-mail : kowalski@math.ethz.ch 